\title{
Synthesis, Characterization and Antimicrobial Evaluation of Novel Imidazole Ureas / Carboxamides Containing Dioxaphospholanes
}

\author{
V. Esther Rani*, CH. Lakshmi Praveena, Y. N. Spoorthy and \\ L. K. Ravindranath. \\ Department of Chemistry, S. K. University, Ananthapuramu, A.P., India. \\ Email:vesther9@gmail.coml
}

\begin{abstract}
New novel derivatives of 1-((6-(4-chlorophenoxy) / (4-bromophenoxy) / (4-nitrophenoxy)-6-oxido-4, 8-dihydro-1H-[1, 3, 2]dioxaphosphepino[5, 6-d]imidazole-1-yl) methyl)-3-phenyl / (p-tolyl) / (4-methoxy phenyl) / (4-chloro phenyl) urea (7a-l as per Scheme-1) and were prepared by condensation of phenyl phosphordichloridates 6(a-c) with 1-((4, 5-bis (hydroxyl methyl)-1H-imidazole-1-yl) methyl)-3-phenyl / (p-tolyl) / (4-methoxy phenyl) / (4-chlorophenyl) urea 5(a-d). The Synthons 5(a-d) were obtained by deprotection of isopropylidene 1-(6, 6-dimethyl-4, 8-dihydro-1H-[1,3] dioxepino[5, 6-d] imidazole-1-yl) methyl)-3-phenyl / (ptolyl) / (4-methoxy phenyl) / (4-chlorophenyl) ureas 4(a-d). The Synthons 4(a-d) were obtained by the condensation of aniline / p-toluidine / 4-methoxyaniline / 4-chloroaniline $\mathbf{3}(\boldsymbol{a}-\boldsymbol{d})$ and 1-(isocyanato methyl)-6, 6 - dimethyl-4, 8 - dihydro - $1 \mathrm{H}$ - [1,3] dioxepino [5, 6-d] imidazole (2). The synthon (2) was obtained from synthon (1). The similar procedure was adapted to prepare $N$-((6- (4-chlorophenoxy) / (4-bromophenoxy) / (4nitrophenoxy) - 6 - oxido - 4, 8-dihyro - $1 \mathrm{H}-[1,3,2]$ dioxaphophepino [5, 6-d] imidazole-1-yl-) methyl) morpholine - 4-carboxamide / piperidine - 1-carboxamide /4-methyl piperazine - 1 -carboxamide (11a-i as per Scheme-2). The products were characterized by $I R,{ }^{1} H N M R,{ }^{13} C N M R,{ }^{31} P N M R$ and elemental analyses. The newly synthesized compounds were subjected to various biological activities viz., antimicrobial.
\end{abstract}

Keywords: Antibacterial, Antifungal, deprotection, imidazole, phenyl phosphorodichloridates.

\section{Introduction}

Imidazole derivetives possess a broad spectrum of pharmalogical activities such as anticonvulsant,

Antiparkinson, monoamine oxidase (MAO) inihibitory activity, anti-bacterial, anti-fungul activity, it also function as dyestuff, catalyst, polymerizing agents, drugs, herbicides and fungicides [1-3]. Imidazole derivatives are valuable vasodialating and vasoconstructing drugs.

The chemistry of phosphorus heterocyclic compounds containing nitrogen has pioneered the application of combinatorial techniques to the development of new pharmaceutical materials with novel properties. Organophosphorus compounds possess significant biological activity against broad spectrum of bacteria, pets, virus, fungicides and plant growth regulators. The organophosphorus heterocyclic compounds chemistry received much attention of chemists in past two decades due to their wide range of applications in the field of the agriculture, medicine and industry. Some organophosphorus compounds have been described in the literature as inhibitors of bacterial, herbicides, insecticides, pesticides, anti-fungal agents, anti-HIV, anti-cancer, anti-viral and anti-inflammatory [4-10].

A good deal of importance was given to dioxaphosphepino ureas / carboxamides and their derivatives in the field of organophosphorus heterocyclic chemistry due to their unique biological applications [11]. In view of the above observations, we synthesized Imidazole possessing dioxaphosphepino ureas / carboxamides and screening for possible biological and pharmacological activities.

\section{Experimental Section}

All the chemicals used in the present investigation were purchased from Sigma-Aldrich Chemicals company, Inc. USA. And used without further purification. TLC was performed on aluminium sheet of silica gel 60F254, E-Merk, Germany using iodine as visualizing agent. Melting point were determined in open capillary tubes on Mel-Temp apparatus and are uncorrected. Column chromatography was performed on silica gel with different solvent systems as eluents to afford the pure compound. The IR Spectra were recorded as $\mathrm{KBr}$ pellets on Perkin-Elmer 1000 units, instruments. All $1 \mathrm{H}$ and ${ }^{13} \mathrm{C}-\mathrm{NMR}$ spectra were recorded on a Varian XL-300 spectrometer operating at $400 \mathrm{MHz}$ for ${ }^{1} \mathrm{H}$-NMR and $75 \mathrm{MHz}$ for ${ }^{13} \mathrm{C}-\mathrm{NMR} .{ }^{31} \mathrm{P}-\mathrm{NMR}$ spectra were recorded on a Varian XL-spectrometer operating at $161.89 \mathrm{MHz}$. The compounds were dissolved in DMSO- $\mathrm{d}_{6}$ and Chemical shifts were referenced to TMS $\left({ }^{1} \mathrm{H}\right.$ and $\left.{ }^{13} \mathrm{C}-\mathrm{NMR}\right)$ and $85 \% \mathrm{H}_{3} \mathrm{PO}_{4}\left({ }^{31} \mathrm{P}-\mathrm{NMR}\right)$. Elemental analysis was recorded on a Carlo Erba 1108 elemental Analyzer, Central Drug Research Institute, Lucknow, India. 


\section{Preparation of Intermediates:}

1.1 Synthesis of phenyl phosphorodichloridates 6(a-c) [12]:<smiles></smiles><smiles>[R]c1ccc(OP(=O)(Cl)Cl)cc1</smiles>

$6(\mathrm{a}-\mathrm{c})$

Phosphorus oxychloride $(15.3 \mathrm{gr}, 0.1 \mathrm{~mole})$ in dry benzene $(60 \mathrm{ml})$ was taken into three-necked flask $(500 \mathrm{ml})$ equipped with dropping funnel and reflux condenser fitted with a calcium chloride guard tube. The flask was heated and stirred by means of hot plate -cum -magnetic stirrer. To this dry triethyl amine (10.1 gr, 0.1 mole) and dry benzene $(50 \mathrm{ml})$ were added slowly and the reaction mixture was stirred for 30 minutes. To this mixture, freshly distilled phenol $(9.4 \mathrm{gr}, 0.1 \mathrm{~mole})$ in dry benzene $(60 \mathrm{ml})$ was added drop wise through the dropping funnel. The addition took about thirty minutes and whole reaction mixture was refluxed with vigorous stirring for 10 hours. The reaction mixture was cooled and the solid tri ethylamine -hydrochloride was filtered off. The solvent from the filtrate was removed under reduced pressure in a rota evaporator. The dark brown liquid remained, was subjected to fractional distillation and the major product distilling at $118-124^{0} \mathrm{C} / 11 \mathrm{~mm}$ was collected as colourless glassy viscous liquid (8.3 gr, 40\%).

Other substituted phenyl phosphorodichlorates $\mathbf{6}(\mathbf{a}-\mathbf{c})$ were prepared by the same procedure [13] by reacting equimolar quantities of phosophorous oxychloride and respective substituted phenols in benzene in the presence of tri ethylamine. The data of these aryl phosphorodichloridates are given in Table -1.

Table-1.1: Phenyl phosphorodichloridate 6(a-c):

\begin{tabular}{|c|c|c|c|c|}
\hline COMP & NAME OF THE DICHLORIDATE & REACTION TIME (hrs) & $\mathrm{Bp}\left({ }^{0} \mathrm{C} / \mathrm{mm}\right)$ & YIELD $(\%)$ \\
\hline $6 \mathrm{a}$ & 4- chloro Phenyl phosphorodichloridate & 9 & $142-145 /$ & 35 \\
\hline $6 \mathrm{~b}$ & 4-bromo Phenyl phosphorodichloridate & 9 & $145-148 / 8$ & 33 \\
\hline $6 c$ & 4-nitro Phenyl phosphorodichloridate & 10 & $134-136 / 6$ & 33 \\
\hline
\end{tabular}<smiles>CC1(C)OCc2ncn(CC(N)=O)c2CO1</smiles>

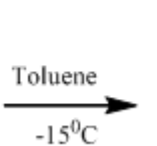<smiles>[R]c1ccc(NC(=O)NCn2cnc(COC(C)(C)C)c2CO)cc1</smiles>

$4(\mathrm{a}-\mathrm{d})$<smiles>CC1(C)OCc2ncn(CN=O)c2CO1</smiles>

2<smiles>[R]c1ccc(N)cc1</smiles>

Deprotection

$\mathrm{CH}_{3} \mathrm{CN} / \mathrm{H}_{2} \mathrm{O}(9 / 1)$ $\mathrm{H}_{3} \mathrm{PW}_{12} \mathrm{O}_{40} \mathrm{nH}_{2} \mathrm{O}(5 \mathrm{~mol} \%)$

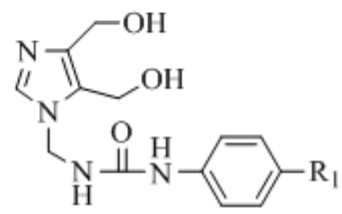

$5(\mathrm{a}-\mathrm{d})$

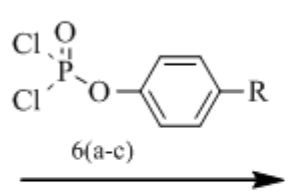<smiles>[R]c1ccc(COP(=O)(OC)OCc2ncn(CNC(=O)Nc3ccc([R])cc3)c2C)cc1</smiles>

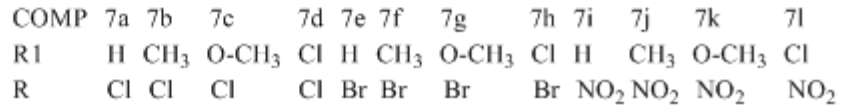

Scheme-I: Synthesis of 1-((6-(4-chlorophenoxy) / (4-bromophenoxy) / (4-nitrophenoxy)-6-oxido-4, 8dihydro-1H-[1, 3, 2]dioxaphosphepino[5, 6-d]imidazole-1-yl) methyl)-3-phenyl / (p-tolyl) / (4-methoxy phenyl) / (4-chloro phenyl) urea 7(a-l) 
<smiles>CC1(C)OCc2ncn(CN=O)c2CO1</smiles>

2

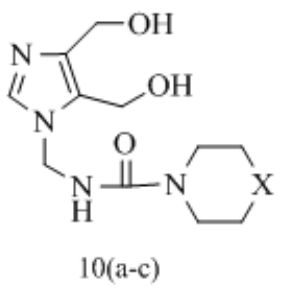

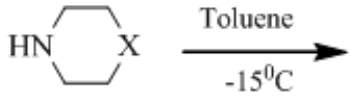

$8(a-c)$

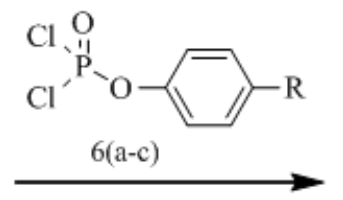<smiles>CC1(C)OCc2ncn(CNC(=O)N3CC[X]CC3)c2CO1</smiles>

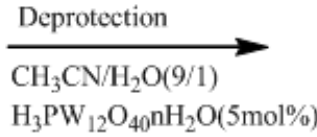<smiles>[X]c1ccc(OP2(=O)OCc3ncn(CNC(=O)N4CCCCC4)c3CO2)cc1</smiles>

$$
\begin{array}{llllllllll}
\text { COMP } & 11 \mathrm{a} & 11 \mathrm{~b} & 11 \mathrm{c} & 1 \mathrm{ld} & 1 \mathrm{le} & 11 \mathrm{f} & 11 \mathrm{~g} & 11 \mathrm{~h} & 11 \mathrm{i} \\
\mathrm{R} & \mathrm{Cl} & \mathrm{Cl} & \mathrm{Cl} & \mathrm{Br} & \mathrm{Br} & \mathrm{Br} & \mathrm{NO}_{2} & \mathrm{NO}_{2} & \mathrm{NO}_{2} \\
\mathrm{X} & \mathrm{O} & \mathrm{CH} 2 & \mathrm{~N}-\mathrm{CH}_{3} & \mathrm{O} & \mathrm{CH}_{2} & \mathrm{~N}-\mathrm{CH}_{3} & \mathrm{O} & \mathrm{CH}_{2} & \mathrm{~N}-\mathrm{CH}_{3}
\end{array}
$$

Scheme-2: Synthesis of N-((6 - (4-chlorophenoxy) / (4-bromophenoxy) / (4-nitrophenoxy) - 6 - oxido - 4, 8dihyro - $1 \mathrm{H}$ - $[1,3,2]$ dioxaphophepino [5, 6-d] imidazole-1-yl-) methyl) morpholine - 4 - carboxamide / piperidine - 1 - carboxamide / 4-methyl piperazine - 1 - carboxamide 11(a-i).

\section{Results And Discussion}

1.2. Synthesis of 1-(isocyanato methyl)-6, 6 - dimethyl-4, 8 - dihydro - 1H - $[1,3]$ dioxepino $[5,6$-d] imidazole (2) [14]:

The 2-(6, 6-dimethyl-4, 8-dihydro-1H-[1, 3] dioxepino[5, 6-d]imidazole-1-yl) acetyl azide (1) (1mmol) dissolved in toluene $(10 \mathrm{~mL})$ was refluxed for $30 \mathrm{~min}$ at $65^{\circ} \mathrm{C}$ under nitrogen atmosphere. After the completion of the reactionas indicated by TLC, the solvent was removed under reduced pressure to get crude 1-(isocyanato methyl) - 6, 6 - dimethyl-4, 8 - dihydro - 1H - [1,3] dioxepino [5, 6-d] imidazole (2). The crude product was purified by column chromatography (60-120 mesh silica gel, eluent: $10 \%$ EtoAc-pet ether), which was recrystallized using dichloromethane and $\mathrm{n}$-hexane, $\mathrm{mp} 139-141^{\circ} \mathrm{C}$, yield $70 \%$.

1.3. Synthesis of 1-(6, 6-dimethyl-4, 8-dihydro-1H-[1, 3] dioxepino [5, 6-d] imidazole-1-yl) methyl) - 3 phenyl / (p-tolyl) / (4-methoxy phenyl) / (4-chlorophenyl) urea 4(a-d):

To the solution of isocyanate (2) (1eq), in aniline (3a) (10 times) dissolved in toluene $(10 \mathrm{~mL})$ was stirred $-15^{\circ} \mathrm{C}$ was refluxed for 16 hours. After completion of the reaction as indicated by TLC, solvent was evaporated under vacuum to give crude residue, purified by column chromatography (60-120 mesh silica gel, eluent: 30\% EtoAc-pet ether). Finally the product compound 1-(6, 6-dimethyl-4, 8-dihydro-1H-[1,3] dioxepino [5, 6-d] imidazole-1-yl) methyl) - 3 - phenyl urea (4a) was purified from aqueous dimethyl formamide with $\mathrm{m} \mathrm{p}$ $166-168^{\circ} \mathrm{C}$, yield $65 \%$.

The similar procedure was adopted to synthesise 4(b-d) by condensing 1-(isocyanato methyl)-6, 6 dimethyl-4, 8 - dihydro - 1H - [1,3] dioxepino [5, 6-d] imidazole (2) with p-toluidine (3b), 4-methoxyaniline (3c) and 4-chloroaniline (3d) respectively.

1.4. Synthesis of 1-((4, 5 - bis (hydroxyl methyl) -1H- imidazole-1-yl) methyl) - 3 -phenyl / (p-tolyl) / (4methoxy phenyl) / (4-chlorophenyl) urea 5(a-d):

The isopropylidenation of 1,2-diols was carried out by a procedure as reported in the literature [15]. A suspension of the 1-(6, 6-dimethyl-4, 8-dihydro-1H-[1, 3] dioxepino [5, 6-d] imidazole-1-yl) methyl) -3- phenyl urea (4a) $(1 \mathrm{~m} \mathrm{~mol})$ in dry acetone and to this $5 \mathrm{~mol} \%$ of phosphotungstic acid was added and the reaction mixture was stirred at room temperature under nitrogen atmosphere for 1 hour The progress of the reaction was monitored by TLC using cyclohexane and ethyl acetate (7:3) solvent mixture as mobile phase. After completion of the reaction, the solvent was removed under reduced pressure. The residue was extracted with dichloromethane $(3 \times 20 \mathrm{ml})$ and water and the combined organic layer was dried with $\mathrm{Na}_{2} \mathrm{SO}_{4}$ and concentrated in vacuum to give the crude product. The crude product was purified by column chromatography on silica gel (60-120 mesh) with $30 \%$ ethyl acetate in cyclohexane as an eluent. The $\mathrm{m} \mathrm{p}$ of (5a) was $182-184^{\circ} \mathrm{C}$ with yield of $74 \%$.

The similar procedure was adopted to synthesise $\mathbf{5}(\mathbf{b}-\mathbf{d})$ from $\mathbf{4}(\mathbf{b}-\mathbf{d})$. 
1.5. Synthesis of 1-((6-(4-chlorophenoxy) / (4-bromophenoxy) / (4-nitrophenoxy)-6-oxido-4, 8-dihydro-1H$[1,3,2]$ dioxaphosphepino $[5,6$-d] imidazole-1-yl) methyl)-3-phenyl / (p-tolyl) / (4-methoxy phenyl) / (4chloro phenyl) urea $7(\mathrm{a}-\mathrm{l})$ :

A solution of 4-chlorophenyl phosphorodichloridate (6a) (0.002 mole) in $25 \mathrm{ml}$ of dry toluene was added drop wise over a period of 20 minutes to a stirred solution of 1-((4, 5-bis (hydroxyl methyl)-1Himidazole-1-yl) methyl)-3-phenyl urea (5a) $(0.002 \mathrm{~mole})$ and triethylamine $(0.004 \mathrm{~mole})$ in $30 \mathrm{ml}$ of dry toluene and $10 \mathrm{ml}$ of tetrahydrofuran at $5^{\circ} \mathrm{c}$. After completion of the addition, the temperature of the reaction mixture was slowly raised to room temperature and stirred for 2 hours. Later the reaction mixture was heated to 50$60^{\circ} \mathrm{C}$ and maintained for 4 hours with stirring. The completion of the reaction was monitored by TLC analysis. Triethyl amine hydrochloric acid was filtered from mixture and solvent was removed under reduced pressure. The residue was washed with water and then recrystallized from aqueous 2-propanol to get pure compound 1((6-(4-chlorophenoxy) - 6 -oxido - 4, 8 - dihydro - 1H - $[1,3,2]$ dioxaphosphepino [5, 6-d] imidazole-1-yl) methyl)-3-phenyl urea (7a), yield $70 \%$, m p $142-144^{\circ} \mathrm{C}$.

The similar procedure was adopted to synthesise $\mathbf{7 ( b - l )}$ by the reaction between $\mathbf{5}(\mathbf{b}-\mathbf{d})$ with 4 chlorophenyl phosphorodichloridate (6a), 4 - bromophenyl phosphorodichloridate (6b) and 4 - nitrophenyl phosphorodichloridate (6c). The structures of newly synthesised of compounds 7(a-l) were established by IR, ${ }^{1} \mathrm{H}-\mathrm{NMR},{ }^{13} \mathrm{C}-\mathrm{NMR},{ }^{31} \mathrm{P}-\mathrm{NMR}$ and elemental analysis. The IR, ${ }^{1} \mathrm{H}-\mathrm{NMR},{ }^{13} \mathrm{C}-\mathrm{NMR},{ }^{31} \mathrm{P}-\mathrm{NMR}$ and analytical data was shown in the Table 1.2-1.6.

1.6. Synthesis of $\mathrm{N}$-((6, 6-dimethyl-4, 8-dihydro-1H-[1, 3] dioxepino [5, 6-d] imidazole-1-yl) methyl) morpholine-4-carboxamide / piperidine-1-carboxamide / 4 - methyl piperazine-1-carboxamide 9(a-c):

To the solution of isocyanate (2) (1eq), in morpholine (8a) (10 times) dissolved in toluene (10mL) was added and heated $-15^{\circ} \mathrm{C}$, the reaction mixture was refluxed for 16 hours. After completion of reaction as indicated by TLC solvent was evaporated under vacuum to give crude residue, purified by column chromatography (60-120 mesh silica gel, eluent: 30\% EtoAc-pet ether) solvent was used as an eluent. Finally the product $\mathrm{N}-((6,6$ - dimethyl - 4, 8 - dihydro - $1 \mathrm{H}$ - [1, 3] dioxepino [5, 6-d] imidazole-1-yl) methyl) morpholine - 4 - carboxamide (9a) was purified from aqueous dimethyl formamide. Yield 65\%, m p 139-142 $\mathrm{C}$. The similar procedure was adopted to synthesise 9(b-c) by condensing 1-(isocyanato methyl) - 6, 6 - dimethyl 4,8 - dihydro - $1 \mathrm{H}-[1,3]$ dioxepino [5, 6-d] imidazole (2) with piperidine (8b) and 1-methylpiperazine (8c) respectively.

1.7. Synthesis of $\mathrm{N}-((4,5$-bis (hydroxyl methyl)-1H -imidazole-1-yl) methyl) morpholine-4-carboxamide / piperidine-1-carboxamide / 4 - methyl piperazine-1-carboxamide 10(a-c):

The isopropylidenation of 1,2-diols was carried out by a procedure as reported in the literature ${ }^{27}$. A suspension of the $\mathrm{N}-((6,6$-dimethyl-4, 8-dihydro-1H-[1, 3] dioxepino [5, 6-d] imidazole-1-yl) methyl) morpholine-4-carboxamide (9a) $(1 \mathrm{~m} \mathrm{~mol})$ in dry acetone and to this $5 \mathrm{~mol} \%$ of phosphotungstic acid was added and the reaction mixture was stirred at room temperature under nitrogen atmosphere for 1 hour The progress of the reaction was monitored by TLC using cyclohexane and ethyl acetate (7:3) solvent mixture as a mobile phase. After completion of the reaction, the solvent was removed under reduced pressure. The residue was extracted with dichloromethane $(3 \times 20 \mathrm{ml})$ and water and the combined organic layer was dried with $\mathrm{Na}_{2} \mathrm{SO}_{4}$ and concentrated in vacuum to give the crude product. The crude product was purified by column chromatography on silica gel (60-120 mesh) with 30\% ethyl acetate in cyclohexane as an eluent. The $\mathrm{m} \mathrm{p}$ of (10a) was $123-126^{\circ} \mathrm{C}$ with yield of $65 \%$.

The similar procedure was adopted to synthesise $\mathbf{1 0}(\mathbf{b}-\mathbf{c})$ from $\mathbf{8}(\mathbf{b}-\mathbf{c})$

1.8. Synthesis of N-((6 - (4-chlorophenoxy) / (4-bromophenoxy) / (4-nitrophenoxy) - 6 - oxido - 4, 8-dihyro - $1 \mathrm{H}$ - $[1,3,2]$ dioxaphophepino [5, 6-d] imidazole-1-yl-) methyl) morpholine - 4 - carboxamide / piperidine - 1 - carboxamide / 4-methyl piperazine - 1 - carboxamide 11(a-i):

A solution of 4-chlorophenyl phosphorodichloridate (6a) $(0.002$ mole) in $25 \mathrm{ml}$ of dry toluene was added drop wise over a period of 20 minutes to a stirred solution of $\mathrm{N}-((4,5$-bis (hydroxyl methyl)-1H imidazole-1-yl) methyl) morpholine-4-carboxamide (10a) (0.002mole) and triethylamine (0.004 mole) in $30 \mathrm{ml}$ of dry toluene and $10 \mathrm{ml}$ of tetrahydrofuran at $5^{\circ} \mathrm{C}$. After completion of the addition, the temperature of the reaction mixture was slowly raised to room temperature and stirred for 2 hours. Later the reaction mixture was heated to $50-60^{\circ} \mathrm{C}$ and maintained for 4 hours with stirring. The completion of the reaction was monitored by TLC analysis. Triethyl amine hydrochloride was filtered from reaction mixture and solvent was removed under reduced pressure. The residue was washed with water and then recrystallized from aqueous 2-propanol to get pure compound $\mathrm{N}-((6$ - (4-chlorophenoxy) - 6 - oxido - 4, 8-dihyro - $1 \mathrm{H}$ - $[1,3,2]$ dioxaphophepino [5, 6-d] imidazole-1-yl-) methyl) morpholine - 4 - carboxamide (11a), yield 70\%, m p 152-155 $\mathrm{C}$. 
The similar procedure was adopted to synthesise 11(b-i) by the reaction between $\mathbf{1 0}(\mathbf{b}-\mathbf{c})$ with 4 - chlorophenyl phosphorodichloridate (6a), 4 - bromophenyl phosphorodichloridate (6b) and 4 - nitrophenyl phosphorodichloridate (6c). The structures of newly synthesised compounds $11(\mathbf{a}-\mathbf{i})$ were established by IR, ${ }^{1} \mathrm{H}-$ NMR, ${ }^{13} \mathrm{C}$-NMR, ${ }^{31} \mathrm{P}$-NMR and elemental analysis. The IR, ${ }^{1} \mathrm{H}$-NMR, ${ }^{13} \mathrm{C}-\mathrm{NMR},{ }^{31} \mathrm{P}$-NMR and analytical data was shown in the Table 1.7-1.11.

Spectral, Physical and analytical data for the compounds:

Table I.2: The IR (KBr) spectra of 1-((6-(4-chlorophenoxy) / (4-bromophenoxy) / (4-nitrophenoxy)-6-oxido-4, 8-dihydro-1H-[1, 3, 2]dioxaphosphepino [5, 6-d] imidazole-1-yl) methyl)-3-phenyl / (p-tolyl) / (4-methoxy phenyl) / (4-chloro phenyl) urea $7(\mathrm{a}-1)\left(\bar{v} / \delta, \mathrm{cm}^{-1}\right)$ :

\begin{tabular}{|c|c|c|c|c|c|c|c|c|c|c|}
\hline COMP & $\mathrm{R}_{1}$ & $\mathrm{R}$ & $\mathrm{N}-\mathrm{H}$ & $\mathrm{Ar}-\mathrm{H}$ & $\mathrm{C}=\mathrm{N}$ & $\mathrm{C}=\mathrm{O}$ & $\mathrm{C}-\mathrm{N}$ & $\mathrm{C}-\mathrm{O}$ & $\mathrm{P}=\mathrm{O}$ & $\mathrm{P}-\mathrm{O}-\mathrm{C}_{(a r o m)}$ \\
\hline $7 \mathrm{a}$ & $\mathrm{H}$ & $\mathrm{Cl}$ & $3418-3384$ & 3100 & 1716 & 1650 & 1416 & 1320 & 1250 & 950 \\
\hline $7 \mathrm{~b}$ & $\mathrm{CH}_{3}$ & $\mathrm{Cl}$ & $3420-3386$ & 3104 & 1718 & 1654 & 1418 & 1324 & 1250 & 950 \\
\hline $7 \mathrm{c}$ & $\mathrm{OCH}_{3}$ & $\mathrm{Cl}$ & $3420-3388$ & 3110 & 1720 & 1660 & 1420 & 1328 & 1250 & 950 \\
\hline $7 \mathrm{~d}$ & $\mathrm{Cl}$ & $\mathrm{Cl}$ & $3422-3390$ & 3120 & 1722 & 1655 & 1424 & 1326 & 1250 & 950 \\
\hline $7 \mathrm{e}$ & $\mathrm{H}$ & $\mathrm{Br}$ & $3418-3384$ & 3125 & 1716 & 1664 & 1410 & 1322 & 1250 & 950 \\
\hline $7 \mathrm{f}$ & $\mathrm{CH}_{3}$ & $\mathrm{Br}$ & $3420-3386$ & 3127 & 1718 & 1660 & 1415 & 1327 & 1250 & 950 \\
\hline $7 \mathrm{~g}$ & $\mathrm{OCH}_{3}$ & $\mathrm{Br}$ & $3420-3388$ & 3129 & 1720 & 1667 & 1426 & 1323 & 1250 & 950 \\
\hline $7 \mathrm{~h}$ & $\mathrm{Cl}$ & $\mathrm{Br}$ & $3422-3390$ & 3130 & 1722 & 1659 & 1417 & 1329 & 1250 & 950 \\
\hline $7 \mathrm{i}$ & $\mathrm{H}$ & $\mathrm{NO}_{2}$ & $3418-3384$ & 3129 & 1720 & 1653 & 1419 & 1320 & 1250 & 950 \\
\hline $7 \mathrm{j}$ & $\mathrm{CH}$ & $\mathrm{NO}_{2}$ & $3420-3386$ & 3130 & 1722 & 1656 & 1423 & 1328 & 1250 & 950 \\
\hline $7 \mathrm{k}$ & $\mathrm{OCH}_{3}$ & $\mathrm{NO}_{2}$ & $3420-3388$ & 3129 & 1720 & 1660 & 1427 & 1325 & 1250 & 950 \\
\hline 71 & $\mathrm{Cl}$ & $\mathrm{NO}_{2}$ & $3422-3390$ & 3130 & 1722 & 1669 & 1423 & 1327 & 1250 & 950 \\
\hline
\end{tabular}

Table1.3: The ${ }^{1} \mathrm{H}$ NMR (400MHz) spectra of 1-((6-(4-chlorophenoxy) / (4-bromophenoxy) / (4-nitrophenoxy)6-oxido-4, 8-dihydro-1H-[1, 3, 2] dioxaphosphepino [5, 6-d]imidazole-1-yl) methyl)-3-phenyl / (p-tolyl) / (4methoxy phenyl) / (4-chloro phenyl) urea $7(\mathrm{a}-1)\left(\delta_{\mathrm{PPM}}\right)$ :

\begin{tabular}{|c|c|c|c|}
\hline COMP & $\mathrm{R}_{1}$ & $\mathrm{R}$ & ${ }^{1} \mathrm{H}-\mathrm{NMR}\left(\mathrm{DMSO}-\mathrm{d}_{6}\right)\left(\boldsymbol{\delta}_{\mathrm{PPM}}\right)$ \\
\hline $7 \mathrm{a}$ & $\mathrm{H}$ & $\mathrm{Cl}$ & $\begin{array}{l}5.23\left(\mathrm{~s}, 4 \mathrm{H}, \text { two } \mathrm{CH}_{2} \text { groups attached to phosphorus moiety), } 5.60\left(\mathrm{~s}, 2 \mathrm{H}, \mathrm{N}-\mathrm{CH}_{2}\right), 6.0(\mathrm{t}, 1 \mathrm{H}, \mathrm{NH} \text { of }\right. \\
\text { urea), } 6.1(\mathrm{~s}, 1 \mathrm{H}, \mathrm{NH} \text { of urea), } 6.89-7.32(\mathrm{~m}, 5 \mathrm{H} \text { of phenyl group), } 7.57(\mathrm{~s}, 1 \mathrm{H}, \mathrm{CH} \text { of imidazole ring) } \\
\text { and } 7.19-7.61(\mathrm{~m}, 4 \mathrm{H} \text { of 4-chlorophenoxy ring). }\end{array}$ \\
\hline $7 \mathrm{~b}$ & $\mathrm{CH}_{3}$ & $\mathrm{Cl}$ & $\begin{array}{l}2.34\left(\mathrm{~s}, 3 \mathrm{H}, \mathrm{CH}_{3} \text { group attached to phenylurea moiety), } 5.23\left(\mathrm{~s}, 4 \mathrm{H}, \text { two } \mathrm{CH}_{2} \text { groups attached to }\right.\right. \\
\text { phosphorus moiety), } 5.60\left(\mathrm{~s}, 2 \mathrm{H}, \mathrm{N}-\mathrm{CH}_{2}\right), 6.0(\mathrm{t}, 1 \mathrm{H}, \mathrm{NH} \text { of urea), } 6.1(\mathrm{~s}, 1 \mathrm{H}, \mathrm{NH} \text { of urea), } 6.90-7.2(\mathrm{~m} \text {, } \\
4 \mathrm{H} \text { of p-tolyl group), } 7.20-7.50(\mathrm{~m}, 4 \mathrm{H} \text { of } 4 \text {-chlorophenoxy ring }) \text { and } 7.57(\mathrm{~s}, 1 \mathrm{H}, \mathrm{CH} \text { of imidazole } \\
\text { ring). }\end{array}$ \\
\hline $7 \mathrm{c}$ & $\mathrm{OCH}_{3}$ & $\mathrm{Cl}$ & $\begin{array}{l}3.80\left(\mathrm{~s}, 3 \mathrm{H}, \mathrm{CH}_{3} \text { group attached to phenylurea moiety), } 5.23\left(\mathrm{~s}, 4 \mathrm{H}, \text { two } \mathrm{CH}_{2} \text { groups attached to }\right.\right. \\
\text { phosphorus moiety), } 5.60\left(\mathrm{~s}, 2 \mathrm{H}, \mathrm{N}-\mathrm{CH}_{2}\right), 6.0(\mathrm{t}, 1 \mathrm{H}, \mathrm{NH} \text { of urea), } 6.1(\mathrm{~s}, 1 \mathrm{H}, \mathrm{NH} \text { of urea), 6.90-7.10 } \\
\text { (m, } 4 \mathrm{H} \text { of } 4-m e t h o x y p h e n y l \text { group), } 6.2-7.5(\mathrm{~m}, 4 \mathrm{H} \text { of } 4 \text {-chlorophenoxy ring) and } 7.57(\mathrm{~s}, 1 \mathrm{H}, \mathrm{CH} \text { of } \\
\text { imidazole ring). }\end{array}$ \\
\hline $7 \mathrm{~d}$ & $\mathrm{Cl}$ & $\mathrm{Cl}$ & $\begin{array}{l}5.23\left(\mathrm{~s}, 4 \mathrm{H}, \text { two } \mathrm{CH}_{2} \text { groups attached to phosphorus moiety), } 5.60\left(\mathrm{~s}, 2 \mathrm{H}, \mathrm{N}-\mathrm{CH}_{2}\right), 6.0(\mathrm{t}, 1 \mathrm{H}, \mathrm{NH} \text { of }\right. \\
\text { urea), } 6.1(\mathrm{~s}, 1 \mathrm{H}, \mathrm{NH} \text { of urea), 7.1-7.3 (m, } 4 \mathrm{H} \text { of 4-chlorophenyl group), } 7.57(\mathrm{~s}, 1 \mathrm{H}, \mathrm{CH} \text { of imidazole } \\
\text { ring) and 7.40-7.70 (m, 4H of 4-chlorophenoxy ring). }\end{array}$ \\
\hline $7 \mathrm{e}$ & $\mathrm{H}$ & $\mathrm{Br}$ & $\begin{array}{l}5.23\left(\mathrm{~s}, 4 \mathrm{H} \text {, two } \mathrm{CH}_{2} \text { groups attached to phosphorus moiety), } 5.60\left(\mathrm{~d}, 2 \mathrm{H}, \mathrm{N}-\mathrm{CH}_{2}\right), 6.0(\mathrm{t}, 1 \mathrm{H}, \mathrm{NH} \text { of }\right. \\
\text { urea), } 6.1(\mathrm{~s}, 1 \mathrm{H}, \mathrm{NH} \text { of urea), 6.89-7.32 (m, } 5 \mathrm{H} \text { of phenyl group), } 7.57(\mathrm{~s}, 1 \mathrm{H}, \mathrm{CH} \text { of imidazole ring) } \\
\text { and } 7.30-7.60(\mathrm{~m}, 4 \mathrm{H} \text { of 4-bromophenoxy ring). }\end{array}$ \\
\hline $7 \mathrm{f}$ & $\mathrm{CH}_{3}$ & $\mathrm{Br}$ & $\begin{array}{l}2.34\left(\mathrm{~s}, 3 \mathrm{H}, \mathrm{CH}_{3} \text { group attached to phenylurea moiety), } 5.23\left(\mathrm{~s}, 4 \mathrm{H}, \text { two } \mathrm{CH}_{2} \text { groups attached to }\right.\right. \\
\text { phosphorus moiety), } 5.60\left(\mathrm{~d}, 2 \mathrm{H}, \mathrm{N}-\mathrm{CH}_{2}\right), 6.0(\mathrm{t}, 1 \mathrm{H}, \mathrm{NH} \text { of urea), } 6.1(\mathrm{~s}, 1 \mathrm{H}, \mathrm{NH} \text { of urea), } 6.90-7.20 \\
(\mathrm{m}, 4 \mathrm{H} \text { of p-tolyl group), } 7.30-7.56(\mathrm{~m}, 4 \mathrm{H} \text { of } 4 \text {-bromophenoxy ring) and } 7.57(\mathrm{~s}, 1 \mathrm{H}, \mathrm{CH} \text { of imidazole } \\
\text { ring). }\end{array}$ \\
\hline $7 \mathrm{~g}$ & $\mathrm{OCH}_{3}$ & $\mathrm{Br}$ & $\begin{array}{l}3.80\left(\mathrm{~s}, 3 \mathrm{H}, \mathrm{CH}_{3} \text { group attached to phenylurea moiety), } 5.23\left(\mathrm{~s}, 4 \mathrm{H}, \text { two } \mathrm{CH}_{2} \text { groups attached to }\right.\right. \\
\text { phosphorus moiety), } 5.60\left(\mathrm{~d}, 2 \mathrm{H}, \mathrm{N}-\mathrm{CH}_{2}\right), 6.0(\mathrm{t}, 1 \mathrm{H}, \mathrm{NH} \text { of urea), } 6.1(\mathrm{~s}, 1 \mathrm{H}, \mathrm{NH} \text { of urea), 6.84-7.43 } \\
\text { (m, 4H of 4-methoxyphenyl group), 7.30-7.54 (m, } 4 \mathrm{H} \text { of 4-bromophenoxy ring) and } 7.57(\mathrm{~s}, 1 \mathrm{H}, \mathrm{CH} \text { of } \\
\text { imidazole ring). }\end{array}$ \\
\hline $7 \mathrm{~h}$ & $\mathrm{Cl}$ & $\mathrm{Br}$ & $\begin{array}{l}5.23\left(\mathrm{~s}, 4 \mathrm{H} \text {, two } \mathrm{CH}_{2} \text { groups attached to phosphorus moiety), } 5.60\left(\mathrm{~d}, 2 \mathrm{H}, \mathrm{N}-\mathrm{CH}_{2}\right), 6.0(\mathrm{t}, 1 \mathrm{H}, \mathrm{NH} \text { of }\right. \\
\text { urea), } 6.1(\mathrm{~s}, 1 \mathrm{H}, \mathrm{NH} \text { of urea), 7.1-7.3 (m, } 4 \mathrm{H} \text { of 4-chlorophenyl group), } 7.57(\mathrm{~s}, 1 \mathrm{H}, \mathrm{CH} \text { of imidazole } \\
\text { ring) and 7.30-7.56 (m, } 4 \mathrm{H} \text { of 4-bromophenoxy ring). }\end{array}$ \\
\hline $7 \mathrm{i}$ & $\mathrm{H}$ & $\mathrm{NO}_{2}$ & $\begin{array}{l}5.23\left(\mathrm{~s}, 4 \mathrm{H}, \text { two } \mathrm{CH}_{2} \text { groups attached to phosphorus moiety), } 5.60\left(\mathrm{~d}, 2 \mathrm{H}, \mathrm{N}-\mathrm{CH}_{2}\right), 6.0(\mathrm{t}, 1 \mathrm{H}, \mathrm{NH} \text { of }\right. \\
\text { urea), } 6.1(\mathrm{~s}, 1 \mathrm{H}, \mathrm{NH} \text { of urea), } 7.57 \text { (s, } 1 \mathrm{H}, \mathrm{CH} \text { of imidazole ring }), 6.90-7.30(\mathrm{~m}, 5 \mathrm{H} \text { of phenyl ring) and } \\
7.36-7.70(\mathrm{~m}, 4 \mathrm{H} \text { of 4-nitrophenoxy group). }\end{array}$ \\
\hline $7 \mathrm{j}$ & $\mathrm{CH}_{3}$ & $\mathrm{NO}_{2}$ & $\begin{array}{l}2.34\left(\mathrm{~s}, 3 \mathrm{H}, \mathrm{CH}_{3} \text { group attached to phenylurea moiety), } 5.23\left(\mathrm{~s}, 4 \mathrm{H}, \text { two } \mathrm{CH}_{2} \text { groups attached to }\right.\right. \\
\text { phosphorus moiety), } 5.60\left(\mathrm{~d}, 2 \mathrm{H}, \mathrm{N}-\mathrm{CH}_{2}\right), 6.0(\mathrm{t}, 1 \mathrm{H}, \mathrm{NH} \text { of urea), } 6.1(\mathrm{~s}, 1 \mathrm{H}, \mathrm{NH} \text { of urea), 6.9-7.2 (m, } \\
4 \mathrm{H} \text { of p-tolyl ring), } 7.57(\mathrm{~s}, 1 \mathrm{H}, \mathrm{CH} \text { of imidazole ring }) \text { and } 7.35-7.70(\mathrm{~m}, 4 \mathrm{H} \text { of 4-nitrophenoxy group). }\end{array}$ \\
\hline $7 \mathrm{k}$ & $\mathrm{OCH}_{3}$ & $\mathrm{NO}_{2}$ & $\begin{array}{l}3.80\left(\mathrm{~s}, 3 \mathrm{H}, \mathrm{CH}_{3} \text { group attached to phenylurea moiety), } 5.23\left(\mathrm{~s}, 4 \mathrm{H}, \text { two } \mathrm{CH}_{2} \text { groups attached to }\right.\right. \\
\text { phosphorus moiety), } 5.60\left(\mathrm{~d}, 2 \mathrm{H}, \mathrm{N}-\mathrm{CH}_{2}\right), 6.0(\mathrm{t}, 1 \mathrm{H}, \mathrm{NH} \text { of urea), } 6.1(\mathrm{~s}, 1 \mathrm{H}, \mathrm{NH} \text { of urea), 6.90-7.10 } \\
\text { (m, } 4 \mathrm{H} \text { of 4-methoxyphenyl ring), } 7.57(\mathrm{~s}, 1 \mathrm{H}, \mathrm{CH} \text { of imidazole ring) and } 7.34-7.72(\mathrm{~m}, 4 \mathrm{H} \text { of } 4- \\
\text { nitrophenoxy group). }\end{array}$ \\
\hline 71 & $\mathrm{Cl}$ & $\mathrm{NO}_{2}$ & $\begin{array}{l}5.23\left(\mathrm{~s}, 4 \mathrm{H}, \text { two } \mathrm{CH}_{2} \text { groups attached to phosphorus moiety), } 5.60\left(\mathrm{~d}, 2 \mathrm{H}, \mathrm{N}-\mathrm{CH}_{2}\right), 6.0(\mathrm{t}, 1 \mathrm{H}, \mathrm{NH} \text { of }\right. \\
\text { urea), } 6.1(\mathrm{~s}, 1 \mathrm{H}, \mathrm{NH} \text { of urea), } 7.57(\mathrm{~s}, 1 \mathrm{H}, \mathrm{CH} \text { of imidazole ring), } 7.10-7.30(\mathrm{~m}, 4 \mathrm{H} \text { of 4-chlorophenyl } \\
\text { ring) and 7.34-7.70 (m, } 4 \mathrm{H} \text { of 4-nitrophenoxy group). }\end{array}$ \\
\hline
\end{tabular}


Table 1.4: The ${ }^{13} \mathrm{C}$ NMR (75MHz) spectra of 1-((6-(4-chlorophenoxy) / (4-bromophenoxy) / (4-nitrophenoxy) 6 - oxido - 4, 8 - dihydro -1H- [1, 3, 2] dioxaphosphepino [5, 6-d] imidazole-1-yl) methyl) -3-phenyl / (p-tolyl) / (4-methoxy phenyl) / (4-chloro phenyl) urea 7(a-l) $\left(\delta_{\mathrm{PPM}}\right)$ :

\begin{tabular}{|c|c|c|c|}
\hline COMP & $\mathrm{R}_{1}$ & $\mathrm{R}$ & ${ }^{13} \mathrm{C}-\mathrm{NMR}\left(\mathrm{DMSO}-\mathrm{d}_{6}\right)\left(\delta_{\mathrm{PPM}}\right)$ \\
\hline $7 \mathrm{a}$ & $\mathrm{H}$ & $\mathrm{Cl}$ & $\begin{array}{l}\text { 137.3, 132.9, 127.7, 63.0, 60.2, 62.4, 154.3, 139.4, 121.6, 128.9, 128.0, 121.6, 148.9, 125.7, } 131.3 \\
\text { and } 126.9 \text { corresponding to } \mathrm{C}_{1}, \mathrm{C}_{2}, \mathrm{C}_{3}, \mathrm{C}_{4}, \mathrm{C}_{5}, \mathrm{C}_{6}, \mathrm{C}_{7}, \mathrm{C}_{8}, \mathrm{C}_{9} \& \mathrm{C}_{13}, \mathrm{C}_{10} \& \mathrm{C}_{12}, \mathrm{C}_{11}, \mathrm{C}_{14}, \mathrm{C}_{18}, \mathrm{C}_{15} \& \\
\mathrm{C}_{19}, \mathrm{C}_{16} \& \mathrm{C}_{18} \text { and } \mathrm{C}_{17} .\end{array}$ \\
\hline $7 b$ & $\mathrm{CH}_{3}$ & $\mathrm{Cl}$ & $\begin{array}{l}\text { 137.3, 132.9, 127.7, 63.0, 60.2, 62.4, 154.3, 136.4, 121.5, 129.2, 136.8, 21.3, } 148.3 \text { and 126.9, } \\
\text { corresponding to } \mathrm{C}_{1}, \mathrm{C}_{2}, \mathrm{C}_{3}, \mathrm{C}_{4}, \mathrm{C}_{5}, \mathrm{C}_{6}, \mathrm{C}_{7}, \mathrm{C}_{8}, \mathrm{C}_{9} \& \mathrm{C}_{13}, \mathrm{C}_{10} \& \mathrm{C}_{12}, \mathrm{C}_{11}, \mathrm{C}_{14}, \mathrm{C}_{15}, \mathrm{C}_{16} \& \mathrm{C}_{20}, \mathrm{C}_{17} \text { \& } \\
\mathrm{C}_{19} \text { and } \mathrm{C}_{18 .}\end{array}$ \\
\hline $7 \mathrm{c}$ & $\mathrm{OCH}_{3}$ & $\mathrm{Cl}$ & $\begin{array}{l}\text { 137.3, 132.9, 127.7, 63.0, 60.2, 62.4, 154.3, 131.7, 119.8, 114.5, 158.9, 55.8, 148.3, 125.7, } 131.3 \text { and } \\
\text { 126.9 corresponding to } \mathrm{C}_{1}, \mathrm{C}_{2}, \mathrm{C}_{3}, \mathrm{C}_{4}, \mathrm{C}_{5}, \mathrm{C}_{6}, \mathrm{C}_{7}, \mathrm{C}_{8}, \mathrm{C}_{9} \& \mathrm{C}_{13}, \mathrm{C}_{10} \& \mathrm{C}_{12}, \mathrm{C}_{11}, \mathrm{C}_{14}, \mathrm{C}_{15}, \mathrm{C}_{16} \& \mathrm{C}_{20} \text {, } \\
\mathrm{C}_{17} \& \mathrm{C}_{19} \text { and } \mathrm{C}_{18} \text {. }\end{array}$ \\
\hline $7 d$ & $\mathrm{Cl}$ & $\mathrm{Cl}$ & $\begin{array}{l}\text { 137.3, 132.9, 127.7, 63.0, 60.2, 62.4, 154.3, 137.5, 120.8, 129.0, 133.3, 148.3, 125.7, } 131.3 \text { and } \\
\text { 126.9 corresponding to } \mathrm{C}_{1}, \mathrm{C}_{2}, \mathrm{C}_{3}, \mathrm{C}_{4}, \mathrm{C}_{5}, \mathrm{C}_{6}, \mathrm{C}_{7}, \mathrm{C}_{8}, \mathrm{C}_{9}, \mathrm{C}_{10} \& \mathrm{C}_{12}, \mathrm{C}_{11}, \mathrm{C}_{13}, \mathrm{C}_{14}, \mathrm{C}_{18}, \mathrm{C}_{15} \& \mathrm{C}_{19}, \mathrm{C}_{16} \\
\& \mathrm{C}_{18} \text { and } \mathrm{C}_{17} \text {. }\end{array}$ \\
\hline $7 \mathrm{e}$ & $\mathrm{H}$ & $\mathrm{Br}$ & $\begin{array}{l}\text { 137.3, 132.9, 127.7, 63.0, 60.2, 62.4, 154.3, 139.4, 121.6, 128.9, 128.0, 149.2, 123.0, 133.0 and } \\
115.7 \text { corresponding to } \mathrm{C}_{1}, \mathrm{C}_{2}, \mathrm{C}_{3}, \mathrm{C}_{4}, \mathrm{C}_{5}, \mathrm{C}_{6}, \mathrm{C}_{7}, \mathrm{C}_{8}, \mathrm{C}_{9}, \mathrm{C}_{10} \& \mathrm{C}_{12}, \mathrm{C}_{11}, \mathrm{C}_{13}, \mathrm{C}_{14}, \mathrm{C}_{18}, \mathrm{C}_{15} \& \mathrm{C}_{19}, \mathrm{C}_{16} \\
\& \mathrm{C}_{18} \text { and } \mathrm{C}_{17} \text {. }\end{array}$ \\
\hline $7 f$ & $\mathrm{CH}_{3}$ & $\mathrm{Br}$ & $\begin{array}{l}\text { 137.3, 132.9, 127.7, 63.0, 60.2, 62.4, 154.3, 136.4, 121.5, 129.2, 136.8, 21.3, 149.2, 123.0, } 133.0 \text { and } \\
115.7 \text { corresponding to } \mathrm{C}_{1}, \mathrm{C}_{2}, \mathrm{C}_{3}, \mathrm{C}_{4}, \mathrm{C}_{5}, \mathrm{C}_{6}, \mathrm{C}_{7}, \mathrm{C}_{8}, \mathrm{C}_{9} \& \mathrm{C}_{13}, \mathrm{C}_{10} \& \mathrm{C}_{12}, \mathrm{C}_{11}, \mathrm{C}_{14}, \mathrm{C}_{15}, \mathrm{C}_{16} \& \mathrm{C}_{20} \text {, } \\
\mathrm{C}_{17} \& \mathrm{C}_{19} \text { and } \mathrm{C}_{18} \text {. }\end{array}$ \\
\hline $7 \mathrm{~g}$ & $\mathrm{OCH}_{3}$ & $\mathrm{Br}$ & $\begin{array}{l}\text { 137.3, 132.9, 127.7, 63.0, 60.2, 62.4, 154.3, 131.7, 119.8, 114.5, 158.9, 55.8, 149.2, 123.0, } 133.0 \text { and } \\
115.7 \text { corresponding to } \mathrm{C}_{1}, \mathrm{C}_{2}, \mathrm{C}_{3}, \mathrm{C}_{4}, \mathrm{C}_{5}, \mathrm{C}_{6}, \mathrm{C}_{7}, \mathrm{C}_{8}, \mathrm{C}_{9} \& \mathrm{C}_{13}, \mathrm{C}_{10} \& \mathrm{C}_{12}, \mathrm{C}_{11}, \mathrm{C}_{14}, \mathrm{C}_{15}, \mathrm{C}_{16} \& \mathrm{C}_{20} \text {, } \\
\mathrm{C}_{17} \& \mathrm{C}_{19} \text { and } \mathrm{C}_{18} \text {. }\end{array}$ \\
\hline $7 \mathrm{~h}$ & $\mathrm{Cl}$ & $\mathrm{Br}$ & $\begin{array}{l}\text { 137.3, 132.9, 127.7, 63.0, 60.2, 62.4, 154.3, 137.5, 120.8, 129.0, 133.3, 149.2, 123.0, } 133.0 \text { and } \\
115.7 \text { corresponding to } \mathrm{C}_{1}, \mathrm{C}_{2}, \mathrm{C}_{3}, \mathrm{C}_{4}, \mathrm{C}_{5}, \mathrm{C}_{6}, \mathrm{C}_{7}, \mathrm{C}_{8}, \mathrm{C}_{9}, \mathrm{C}_{10} \& \mathrm{C}_{12}, \mathrm{C}_{11}, \mathrm{C}_{13}, \mathrm{C}_{14}, \mathrm{C}_{18}, \mathrm{C}_{15} \text { \& } \mathrm{C}_{19}, \mathrm{C}_{16} \\
\& \mathrm{C}_{18} \text { and } \mathrm{C}_{17} \text {. }\end{array}$ \\
\hline $7 \mathrm{i}$ & $\mathrm{H}$ & $\mathrm{NO}_{2}$ & $\begin{array}{l}137.3,132.9,127.7,63.0,60.2,62.4,154.3,139.4,121.6,128.9,128.0,, 156.3,121.9,126.3 \text { and } \\
140.5 \text { corresponding to } \mathrm{C}_{1}, \mathrm{C}_{2}, \mathrm{C}_{3}, \mathrm{C}_{4}, \mathrm{C}_{5}, \mathrm{C}_{6}, \mathrm{C}_{7}, \mathrm{C}_{8}, \mathrm{C}_{9}, \mathrm{C}_{10} \& \mathrm{C}_{12}, \mathrm{C}_{11}, \mathrm{C}_{13}, \mathrm{C}_{14}, \mathrm{C}_{18}, \mathrm{C}_{15} \text { \& } \mathrm{C}_{19}, \mathrm{C}_{16} \\
\& \mathrm{C}_{18} \text { and } \mathrm{C}_{17} \text {. }\end{array}$ \\
\hline $7 \mathrm{j}$ & $\mathrm{CH}_{3}$ & $\mathrm{NO}_{2}$ & $\begin{array}{l}\text { 137.3, 132.9, 127.7, 63.0, 60.2, 62.4, 154.3, 136.4, 121.5, 129.2, 136.8, 129.2, 121.5, 21.3, 156.3, } \\
121.9,126.3 \text { and } 140.5 \text { corresponding to } \mathrm{C}_{1}, \mathrm{C}_{2}, \mathrm{C}_{3}, \mathrm{C}_{4}, \mathrm{C}_{5}, \mathrm{C}_{6}, \mathrm{C}_{7}, \mathrm{C}_{8}, \mathrm{C}_{9} \& \mathrm{C}_{13}, \mathrm{C}_{10} \& \mathrm{C}_{12}, \mathrm{C}_{11}, \mathrm{C}_{14} \text {, } \\
\mathrm{C}_{15}, \mathrm{C}_{16} \& \mathrm{C}_{20}, \mathrm{C}_{17} \& \mathrm{C}_{19} \text { and } \mathrm{C}_{18} \text {. }\end{array}$ \\
\hline $7 \mathrm{k}$ & $\mathrm{OCH}_{3}$ & $\mathrm{NO}_{2}$ & $\begin{array}{l}\text { 137.3, 132.9, 127.7, 63.0, 60.2, 62.4, 154.3, 131.7, 119.8, 114.5, 158.9, 55.8, 156.3, 121.9, } 126.3 \text { and } \\
\text { 140.5, corresponding to } \mathrm{C}_{1}, \mathrm{C}_{2}, \mathrm{C}_{3}, \mathrm{C}_{4}, \mathrm{C}_{5}, \mathrm{C}_{6}, \mathrm{C}_{7}, \mathrm{C}_{8}, \mathrm{C}_{9} \& \mathrm{C}_{13}, \mathrm{C}_{10} \& \mathrm{C}_{12}, \mathrm{C}_{11}, \mathrm{C}_{14}, \mathrm{C}_{15}, \mathrm{C}_{16} \& \mathrm{C}_{20} \text {, } \\
\mathrm{C}_{17} \& \mathrm{C}_{19} \text { and } \mathrm{C}_{18} \text {. }\end{array}$ \\
\hline 71 & $\mathrm{Cl}$ & $\mathrm{NO}_{2}$ & $\begin{array}{l}137.3,132.9,127.7,63.0,60.2,62.4,154.3,137.5,120.8,129.0,133.3,156.3,121.9,126.3 \text { and } \\
140.5 \text { corresponding to } C_{1}, C_{2}, C_{3}, C_{4}, C_{5}, C_{6}, C_{7}, C_{8}, C_{9}, C_{10} \& C_{12}, C_{11}, C_{13}, C_{14}, C_{18}, C_{15} \& C_{19}, C_{16} \\
\& C_{18} \text { and } C_{17} \text {. }\end{array}$ \\
\hline
\end{tabular}

Table 1.5: The ${ }^{31} \mathrm{P}$ NMR (161.89MHz) spectra of 1-((6-(4-chlorophenoxy) / (4-bromophenoxy) / (4nitrophenoxy) - 6 - oxido - 4, 8 - dihydro - $1 \mathrm{H}$ - $[1,3,2]$ dioxaphosphepino [5, 6-d] imidazole-1-yl) methyl) -3phenyl / (4-chloro phenyl) urea (7a, 7d, 7e, 7h, 7i and 7l) $\left(\delta_{\text {PPM }}\right)$ :

\begin{tabular}{|c|c|c|c|}
\hline COMP & $\mathrm{R}_{1}$ & $\mathrm{R}$ & ${ }^{31} \mathrm{P}-\mathrm{NMR}\left(\mathrm{DMSO}_{\mathrm{d}}\right)\left(\delta_{\mathrm{PPM}}\right)$ \\
\hline $7 \mathrm{a}$ & $\mathrm{H}$ & $\mathrm{Cl}$ & -7.60 and -11.20 \\
\hline $7 \mathrm{~d}$ & $\mathrm{Cl}$ & $\mathrm{Cl}$ & -7.40 \\
\hline $7 \mathrm{e}$ & $\mathrm{H}$ & $\mathrm{Br}$ & -7.90 and -11.50 \\
\hline $7 \mathrm{~h}$ & $\mathrm{Cl}$ & $\mathrm{Br}$ & -7.70 \\
\hline $7 \mathrm{i}$ & $\mathrm{H}$ & $\mathrm{NO}_{2}$ & -7.20 and -10.50 \\
\hline 71 & $\mathrm{Cl}$ & $\mathrm{NO}_{2}$ & -7.10 \\
\hline
\end{tabular}

Table 1.6: Pysical and Analytical data of 1-((6-(4-chlorophenoxy) / (4-bromophenoxy) / (4-nitrophenoxy)-6oxido-4, 8-dihydro-1H-[1, 3, 2]dioxaphosphepino [5, 6-d] imidazole-1-yl) methyl)-3-phenyl / (p-tolyl) / (4methoxy phenyl) / (4-chloro phenyl) urea 7(a-1):

\begin{tabular}{|c|c|c|c|c|c|c|c|}
\hline \multirow[t]{2}{*}{ COMP } & \multirow[t]{2}{*}{$\begin{array}{l}\text { MOLECULAR } \\
\text { FORMULA }\end{array}$} & \multirow[t]{2}{*}{$\operatorname{MP}\left({ }^{0} \mathrm{C}\right)$} & \multirow[t]{2}{*}{$\begin{array}{c}\text { YIELD } \\
(\%)\end{array}$} & \multicolumn{4}{|c|}{$\begin{array}{l}\text { ELEMENTAL } \\
\text { ANALYSS }(\%)\end{array}$} \\
\hline & & & & & OUND & & ALC \\
\hline $7 \mathrm{a}$ & $\mathrm{C}_{19} \mathrm{H}_{18} \mathrm{ClN}_{4} \mathrm{O}_{5} \mathrm{P}$ & $142-144$ & 70 & $\begin{array}{l}\mathrm{C} \\
\mathrm{H} \\
\mathrm{Cl} \\
\mathrm{N} \\
\mathrm{P}\end{array}$ & $\begin{array}{r}50.05, \\
3.54, \\
7.10, \\
11.88, \\
6.20 .\end{array}$ & $\begin{array}{l}\mathrm{C} \\
\mathrm{H} \\
\mathrm{Cl} \\
\mathrm{N} \\
\mathrm{P}\end{array}$ & $\begin{array}{r}50.85, \\
4.04, \\
7.90, \\
12.48, \\
6.90 .\end{array}$ \\
\hline $7 b$ & $\mathrm{C}_{20} \mathrm{H}_{20} \mathrm{ClN}_{4} \mathrm{O}_{5} \mathrm{P}$ & $163-165$ & 70 & $\begin{array}{l}\mathrm{C} \\
\mathrm{H} \\
\mathrm{Cl} \\
\mathrm{N} \\
\mathrm{P}\end{array}$ & $\begin{array}{c}51.10, \\
3.86, \\
6.86, \\
11.41, \\
5.99 .\end{array}$ & $\begin{array}{l}\mathrm{C} \\
\mathrm{H} \\
\mathrm{Cl} \\
\mathrm{N} \\
\mathrm{P}\end{array}$ & $\begin{array}{r}51.90, \\
4.36, \\
7.66, \\
12.11, \\
6.69 .\end{array}$ \\
\hline $7 \mathrm{c}$ & $\mathrm{C}_{20} \mathrm{H}_{20} \mathrm{ClN}_{4} \mathrm{O}_{6} \mathrm{P}$ & $179-181$ & 75 & $\begin{array}{l}\mathrm{C} \\
\mathrm{H}\end{array}$ & $\begin{array}{r}49.37 \\
3.71\end{array}$ & $\begin{array}{l}\mathrm{C} \\
\mathrm{H}\end{array}$ & $\begin{array}{r}50.17, \\
4.21,\end{array}$ \\
\hline
\end{tabular}




\begin{tabular}{|c|c|c|c|c|c|c|}
\hline & & & & $\begin{array}{l}\mathrm{Cl} \\
\mathrm{N} \\
\mathrm{P}\end{array}$ & $\begin{array}{l}6.60, \\
11.10, \\
5.77 .\end{array}$ & $\begin{array}{ll}\mathrm{Cl} & 7.40, \\
\mathrm{~N} & 11.70, \\
\mathrm{P} & 6.47 .\end{array}$ \\
\hline $7 d$ & $\mathrm{C}_{19} \mathrm{H}_{17} \mathrm{Cl}_{2} \mathrm{~N}_{4} \mathrm{O}_{5} \mathrm{P}$ & $160-162$ & 72 & $\begin{array}{l}\mathrm{C} \\
\mathrm{H} \\
\mathrm{Cl} \\
\mathrm{N} \\
\mathrm{P}\end{array}$ & $\begin{array}{r}46.42, \\
3.05, \\
13.87, \\
10.99, \\
5.71 .\end{array}$ & $\begin{array}{lr}\mathrm{C} & 47.22, \\
\mathrm{H} & 3.55, \\
\mathrm{Cl} & 14.67, \\
\mathrm{~N} & 11.59, \\
\mathrm{P} & 6.41 .\end{array}$ \\
\hline $7 e$ & $\mathrm{C}_{19} \mathrm{H}_{18} \mathrm{BrN}_{4} \mathrm{O}_{5} \mathrm{P}$ & $161-163$ & 70 & $\begin{array}{l}\mathrm{C} \\
\mathrm{H} \\
\mathrm{Br} \\
\mathrm{N} \\
\mathrm{P}\end{array}$ & $\begin{aligned} 45.47, \\
3.18, \\
15.60, \\
10.76, \\
5.58 .\end{aligned}$ & $\begin{array}{lr}\mathrm{C} & 46.27, \\
\mathrm{H} & 3.68, \\
\mathrm{Br} & 16.20, \\
\mathrm{~N} & 11.36, \\
\mathrm{P} & 6.28 .\end{array}$ \\
\hline $7 f$ & $\mathrm{C}_{20} \mathrm{H}_{20} \mathrm{BrN}_{4} \mathrm{O}_{5} \mathrm{P}$ & $157-159$ & 65 & $\begin{array}{l}\mathrm{C} \\
\mathrm{H} \\
\mathrm{Br} \\
\mathrm{N} \\
\mathrm{P}\end{array}$ & $\begin{array}{r}46.55, \\
3.47, \\
15.15, \\
10.44, \\
5.41 .\end{array}$ & $\begin{array}{lr}\mathrm{C} & 47.35, \\
\mathrm{H} & 3.97, \\
\mathrm{Br} & 15.75, \\
\mathrm{~N} & 11.04, \\
\mathrm{P} & 6.11 .\end{array}$ \\
\hline $7 g$ & $\mathrm{C}_{20} \mathrm{H}_{20} \mathrm{BrN}_{4} \mathrm{O}_{6} \mathrm{P}$ & $178-180$ & 60 & $\begin{array}{l}\mathrm{C} \\
\mathrm{H} \\
\mathrm{Br} \\
\mathrm{N} \\
\mathrm{P}\end{array}$ & $\begin{array}{r}45.11, \\
3.35, \\
14.67, \\
10.11, \\
5.22 .\end{array}$ & $\begin{array}{lr}\mathrm{C} & 45.91, \\
\mathrm{H} & 3.85, \\
\mathrm{Br} & 15.27, \\
\mathrm{~N} & 10.71, \\
\mathrm{P} & 5.92 .\end{array}$ \\
\hline $7 \mathrm{~h}$ & $\mathrm{C}_{19} \mathrm{H}_{17} \mathrm{BrClN}_{4} \mathrm{O}_{5} \mathrm{P}$ & $185-187$ & 64 & $\begin{array}{l}\mathrm{C} \\
\mathrm{H} \\
\mathrm{Br} \\
\mathrm{Cl} \\
\mathrm{N} \\
\mathrm{P}\end{array}$ & $\begin{array}{r}42.45, \\
2.75, \\
14.54, \\
5.92, \\
10.02, \\
5.17 .\end{array}$ & $\begin{array}{lr}\mathrm{C} & 43.25, \\
\mathrm{H} & 3.25, \\
\mathrm{Br} & 15.14, \\
\mathrm{Cl} & 6.72, \\
\mathrm{~N} & 10.62, \\
\mathrm{P} & 5.87 .\end{array}$ \\
\hline $7 \mathrm{i}$ & $\mathrm{C}_{19} \mathrm{H}_{18} \mathrm{~N}_{5} \mathrm{O}_{7} \mathrm{P}$ & $173-175$ & 60 & $\begin{array}{l}\mathrm{C} \\
\mathrm{H} \\
\mathrm{N} \\
\mathrm{P}\end{array}$ & $\begin{array}{r}48.88, \\
3.45, \\
14.65, \\
6.04 .\end{array}$ & $\begin{array}{lr}\mathrm{C} & 49.68, \\
\mathrm{H} & 3.95, \\
\mathrm{~N} & 15.25, \\
\mathrm{P} & 6.74 . \\
\end{array}$ \\
\hline $7 \mathrm{j}$ & $\mathrm{C}_{20} \mathrm{H}_{20} \mathrm{~N}_{5} \mathrm{O}_{7} \mathrm{P}$ & $165-167$ & 65 & $\begin{array}{l}\mathrm{C} \\
\mathrm{H} \\
\mathrm{N} \\
\mathrm{P}\end{array}$ & $\begin{array}{r}49.94, \\
4.11, \\
14.19, \\
5.94 . \\
\end{array}$ & $\begin{array}{lr}\mathrm{C} & 50.74, \\
\mathrm{H} & 4.61, \\
\mathrm{~N} & 14.79, \\
\mathrm{P} & 6.54 . \\
\end{array}$ \\
\hline $7 \mathrm{k}$ & $\mathrm{C}_{20} \mathrm{H}_{20} \mathrm{~N}_{5} \mathrm{O}_{8} \mathrm{P}$ & $193-195$ & 65 & $\begin{array}{l}\mathrm{C} \\
\mathrm{H} \\
\mathrm{N} \\
\mathrm{P}\end{array}$ & $\begin{array}{r}48.29, \\
3.62, \\
13.71, \\
5.63 .\end{array}$ & $\begin{array}{r}49.09, \\
4.12, \\
14.31, \\
6.33 .\end{array}$ \\
\hline 71 & $\mathrm{C}_{19} \mathrm{H}_{17} \mathrm{ClN}_{5} \mathrm{O}_{7} \mathrm{P}$ & $207-209$ & 70 & $\begin{array}{l}\mathrm{C} \\
\mathrm{H} \\
\mathrm{Cl} \\
\mathrm{N} \\
\mathrm{P}\end{array}$ & $\begin{array}{r}45.41, \\
2.97, \\
6.38, \\
13.58, \\
5.67 .\end{array}$ & $\begin{array}{lr}\mathrm{C} & 46.21, \\
\mathrm{H} & 3.47, \\
\mathrm{Cl} & 7.18, \\
\mathrm{~N} & 14.18, \\
\mathrm{P} & 6.27 .\end{array}$ \\
\hline
\end{tabular}

Table 1.7: The IR (KBr) spectra of N-((6 -(4-chlorophenoxy) / (4-bromophenoxy) / (4-nitrophenoxy)-6-oxido-4, 8-dihyro-1H-[1, 3, 2] dioxaphophepino [5, 6-d] imidazole-1-yl-) methyl) morpholine - 4 - carboxamide / piperidine - 1 - carboxamide / 4-methyl piperazine - 1 - carboxamide $11(\mathrm{a}-\mathrm{i})\left(\overline{\mathrm{v}} / \delta, \mathrm{cm}^{-1}\right)$ :

\begin{tabular}{|c|c|c|c|c|c|c|c|c|c|}
\hline COMP & $\mathrm{R}$ & $\mathrm{X}$ & $\mathrm{N}-\mathrm{H}$ & $\mathrm{C}=\mathrm{O}$ & $\mathrm{C}=\mathrm{N}$ & $\mathrm{C}-\mathrm{N}$ & $\mathrm{C}-\mathrm{O}$ & $\mathrm{P}=\mathrm{O}$ & $\mathrm{P}-\mathrm{O}-\mathrm{C}_{(\mathrm{arom})}$ \\
\hline $11 \mathrm{a}$ & $\mathrm{Cl}$ & $\mathrm{O}$ & 3418 & 1650 & 1616 & 1416 & 1320 & 1250 & 950 \\
\hline $11 \mathrm{~b}$ & $\mathrm{Cl}$ & $\mathrm{CH}_{2}$ & 3420 & 1655 & 1618 & 1418 & 1325 & 1245 & 955 \\
\hline $11 \mathrm{c}$ & $\mathrm{Cl}$ & $\mathrm{N}^{-\mathrm{CH}_{3}}$ & 3420 & 1656 & 1620 & 1420 & 1323 & 1240 & 960 \\
\hline $11 \mathrm{~d}$ & $\mathrm{Br}$ & $\mathrm{O}$ & 3422 & 1653 & 1622 & 1423 & 1326 & 1255 & 954 \\
\hline $11 \mathrm{e}$ & $\mathrm{Br}$ & $\mathrm{CH}_{2}$ & 3418 & 1657 & 1616 & 1414 & 1328 & 1260 & 940 \\
\hline $11 \mathrm{f}$ & $\mathrm{Br}$ & $\mathrm{N}-C H_{3}$ & 3420 & 1660 & 1618 & 1419 & 1327 & 1259 & 948 \\
\hline $11 \mathrm{~g}$ & $\mathrm{NO}_{2}$ & $\mathrm{O}$ & 3420 & 1665 & 1620 & 1420 & 1315 & 1264 & 958 \\
\hline $11 \mathrm{~h}$ & $\mathrm{NO}_{2}$ & $\mathrm{CH}_{2}$ & 3422 & 1663 & 1622 & 1424 & 1319 & 1269 & 963 \\
\hline $11 \mathrm{i}$ & $\mathrm{NO}_{2}$ & $\mathrm{~N}_{-}-\mathrm{CH}_{3}$ & 3418 & 1664 & 1624 & 1425 & 1323 & 1254 & 968 \\
\hline
\end{tabular}


Table 1.8: The ${ }^{1} \mathrm{H}$ NMR (400MHz) spectra of N-((6 - (4-chlorophenoxy) / (4-bromophenoxy) / (4nitrophenoxy) - 6 - oxido - 4, 8-dihyro - $1 \mathrm{H}$ - [1,3,2] dioxaphophepino [5, 6-d] imidazole-1-yl-) methyl) morpholine - 4 - carboxamide / piperidine - 1 - carboxamide / 4-methyl piperazine - 1 - carboxamide 11(a-i) $(\delta$

\begin{tabular}{|c|c|c|c|}
\hline COMP & $\mathrm{R}$ & $\mathrm{X}$ & ${ }^{1} \mathrm{H}-\mathrm{NMR}\left(\mathrm{DMSO}-\mathrm{d}_{6}\right)\left(\delta_{\mathrm{PPM}}\right)$ \\
\hline $11 \mathrm{a}$ & $\mathrm{Cl}$ & $\mathrm{O}$ & $\begin{array}{l}3.31\left(\mathrm{t}, 4 \mathrm{H}, \mathrm{N}-\mathrm{CH}_{2} \text { of morpholine ring }\right), 3.65\left(\mathrm{t}, 4 \mathrm{H},-\mathrm{CH}_{2}-\mathrm{O} \text { of morpholine ring }\right), 5.23(\mathrm{~s}, 4 \mathrm{H} \text {, two } \\
\mathrm{CH}_{2} \text { groups attached to phosphorus moiety), } 5.60\left(\mathrm{~d}, 2 \mathrm{H}, \mathrm{N}-\mathrm{CH}_{2}\right), 6.0(\mathrm{~s}, 1 \mathrm{H}, \mathrm{NH}-\text {-of carboxamide } \\
\text { moiety), } 6.89-7.32(\mathrm{~m}, 4 \mathrm{H} \text {, of phenoxy group) and } 7.57(\mathrm{~s}, 1 \mathrm{H} \text { of imidazole ring). }\end{array}$ \\
\hline $11 \mathrm{~b}$ & $\mathrm{Cl}$ & $\mathrm{CH}_{2}$ & $\begin{array}{l}\text { 1.53-1.59 }\left(\mathrm{m}, 6 \mathrm{H}, \mathrm{CH}_{2} \text { piperidine of carboxamide), } 3.77\left(\mathrm{t}, 4 \mathrm{H}, \mathrm{N}-\mathrm{CH}_{2} \text { piperidine of carboxamide }\right) \text {, }\right. \\
5.23\left(\mathrm{~s}, 4 \mathrm{H} \text {, two } \mathrm{CH}_{2} \text { groups attached to phosphorus moiety), } 5.60\left(\mathrm{~d}, 2 \mathrm{H}, \mathrm{N}-\mathrm{CH}_{2}\right), 6.0(\mathrm{~s}, 1 \mathrm{H}, \mathrm{NH}-\right. \\
\text { of carboxamide moiety), 6.89-7.32 }(\mathrm{m}, 4 \mathrm{H} \text {, of phenoxy group) and } 7.57(\mathrm{~s}, 1 \mathrm{H} \text { of imidazole ring). }\end{array}$ \\
\hline $11 \mathrm{c}$ & $\mathrm{Cl}$ & $\mathrm{N}-\mathrm{CH}_{3}$ & $\begin{array}{l}2.10\left(\mathrm{~s}, 3 \mathrm{H}, \mathrm{N}-\mathrm{CH}_{3}\right), 2.27\left(\mathrm{t}, 4 \mathrm{H}, \mathrm{CH}_{2}-\mathrm{N} \text { piperazine of carboxamide), } 3.40\left(\mathrm{t}, 4 \mathrm{H}, \mathrm{N}-\mathrm{CH}_{2} \text { piperazine }\right.\right. \\
\text { of carboxamide), } 5.23\left(\mathrm{~s}, 4 \mathrm{H}, \text { two } \mathrm{CH}_{2} \text { groups attached to phosphorus moiety), } 5.60(\mathrm{~d}, 2 \mathrm{H}, \mathrm{N}-\right. \\
\left.\mathrm{CH}_{2}\right), 6.0(\mathrm{~s}, 1 \mathrm{H}, \mathrm{NH}-\text { of carboxamide moiety), } 6.89-7.32(\mathrm{~m}, 4 \mathrm{H} \text {, of phenoxy group) and } 7.57(\mathrm{~s}, 1 \mathrm{H} \\
\text { of imidazole ring). }\end{array}$ \\
\hline $11 d$ & $\mathrm{Br}$ & $\mathrm{O}$ & $\begin{array}{l}3.31\left(\mathrm{t}, 4 \mathrm{H}, \mathrm{N}-\mathrm{CH}_{2} \text { of morpholine ring), } 3.65\left(\mathrm{t}, 4 \mathrm{H},-\mathrm{CH}_{2}-\mathrm{O} \text { of morpholine ring), } 5.23(\mathrm{~s}, 4 \mathrm{H} \text {, two }\right.\right. \\
\mathrm{CH}_{2} \text { groups attached to phosphorus moiety), } 5.60\left(\mathrm{~d}, 2 \mathrm{H}, \mathrm{N}-\mathrm{CH}_{2}\right), 6.0(\mathrm{~s}, 1 \mathrm{H}, \mathrm{NH} \text {-of carboxamide } \\
\text { moiety), } 6.80-7.23(\mathrm{~m}, 4 \mathrm{H} \text {, of phenoxy group) and } 7.57(\mathrm{~s}, 1 \mathrm{H} \text { of imidazole ring). }\end{array}$ \\
\hline $11 \mathrm{e}$ & $\mathrm{Br}$ & $\mathrm{CH}_{2}$ & $\begin{array}{l}\text { 1.53-1.59 }\left(\mathrm{m}, 6 \mathrm{H}, \mathrm{CH}_{2} \text { piperidine of carboxamide), } 3.77\left(\mathrm{t}, 4 \mathrm{H}, \mathrm{N}-\mathrm{CH}_{2} \text { piperidine of carboxamide), }\right.\right. \\
5.23\left(\mathrm{~s}, 4 \mathrm{H} \text {, two } \mathrm{CH}_{2} \text { groups attached to phosphorus moiety), } 5.60\left(\mathrm{~d}, 2 \mathrm{H}, \mathrm{N}-\mathrm{CH}_{2}\right), 6.0(\mathrm{~s}, 1 \mathrm{H}, \mathrm{NH}-\right. \\
\text { of carboxamide moiety), } 6.80-7.23(\mathrm{~m}, 4 \mathrm{H} \text {, of phenoxy group) and } 7.57(\mathrm{~s}, 1 \mathrm{H} \text { of imidazole ring). }\end{array}$ \\
\hline $11 \mathrm{f}$ & $\mathrm{Br}$ & $\mathrm{N}-\mathrm{CH}_{3}$ & $\begin{array}{l}2.10\left(\mathrm{~s}, 3 \mathrm{H}, \mathrm{N}-\mathrm{CH}_{3}\right), 2.27\left(\mathrm{t}, 4 \mathrm{H}, \mathrm{CH}_{2}-\mathrm{N} \text { piperazine of carboxamide), } 3.40\left(\mathrm{t}, 4 \mathrm{H}, \mathrm{N}-\mathrm{CH}_{2} \text { piperazine }\right.\right. \\
\text { of carboxamide), } 5.23\left(\mathrm{~s}, 4 \mathrm{H}, \text { two } \mathrm{CH}_{2} \text { groups attached to phosphorus moiety), } 5.60(\mathrm{~d}, 2 \mathrm{H}, \mathrm{N}-\right. \\
\left.\mathrm{CH}_{2}\right), 6.0(\mathrm{~s}, 1 \mathrm{H}, \mathrm{NH}-\text { of carboxamide moiety), } 6.80-7.23(\mathrm{~m}, 4 \mathrm{H} \text {, of phenoxy group) and } 7.57(\mathrm{~s}, 1 \mathrm{H} \\
\text { of imidazole ring). }\end{array}$ \\
\hline $11 \mathrm{~g}$ & $\mathrm{NO}_{2}$ & $\mathrm{O}$ & $\begin{array}{l}3.31\left(\mathrm{t}, 4 \mathrm{H}, \mathrm{N}-\mathrm{CH}_{2} \text { of morpholine ring), } 3.65\left(\mathrm{t}, 4 \mathrm{H},-\mathrm{CH}_{2}-\mathrm{O} \text { of morpholine ring), } 5.23(\mathrm{~s}, 4 \mathrm{H} \text {, two }\right.\right. \\
\mathrm{CH}_{2} \text { groups attached to phosphorus moiety), } 5.60\left(\mathrm{~d}, 2 \mathrm{H}, \mathrm{N}-\mathrm{CH}_{2}\right), 6.0(\mathrm{~s}, 1 \mathrm{H}, \mathrm{NH} \text {-of carboxamide } \\
\text { moiety), } 7.57(\mathrm{~s}, 1 \mathrm{H} \text { of imidazole ring) and } 7.34-8.09(\mathrm{~m}, 4 \mathrm{H}, \text { of phenoxy group). }\end{array}$ \\
\hline $11 \mathrm{~h}$ & $\mathrm{NO}_{2}$ & $\mathrm{CH}_{2}$ & $\begin{array}{l}\text { 1.53-1.59 }\left(\mathrm{m}, 6 \mathrm{H}, \mathrm{CH}_{2} \text { piperidine of carboxamide), } 3.77\left(\mathrm{t}, 4 \mathrm{H}, \mathrm{N}-\mathrm{CH}_{2} \text { piperidine of carboxamide), }\right.\right. \\
5.23\left(\mathrm{~s}, 4 \mathrm{H} \text {, two } \mathrm{CH}_{2} \text { groups attached to phosphorus moiety), } 5.60\left(\mathrm{~d}, 2 \mathrm{H}, \mathrm{N}-\mathrm{CH}_{2}\right), 6.0(\mathrm{~s}, 1 \mathrm{H}, \mathrm{NH}-\right. \\
\text { of carboxamide moiety), } 7.57(\mathrm{~s}, 1 \mathrm{H} \text { of imidazole ring) and } 7.34-8.09(\mathrm{~m}, 4 \mathrm{H} \text {, of phenoxy group). }\end{array}$ \\
\hline $11 \mathrm{i}$ & $\mathrm{NO}_{2}$ & $\mathrm{~N}-\mathrm{CH}_{3}$ & $\begin{array}{l}2.10\left(\mathrm{~s}, 3 \mathrm{H}, \mathrm{N}-\mathrm{CH}_{3}\right), 2.27\left(\mathrm{t}, 4 \mathrm{H}, \mathrm{CH}_{2}-\mathrm{N} \text { piperazine of carboxamide), } 3.40\left(\mathrm{t}, 4 \mathrm{H}, \mathrm{N}-\mathrm{CH}_{2} \text { piperazine }\right.\right. \\
\text { of carboxamide), } 5.23\left(\mathrm{~s}, 4 \mathrm{H}, \text { two } \mathrm{CH}_{2} \text { groups attached to phosphorus moiety), } 5.60(\mathrm{~d}, 2 \mathrm{H}, \mathrm{N}-\right. \\
\left.\mathrm{CH}_{2}\right), 6.0(\mathrm{~s}, 1 \mathrm{H}, \mathrm{NH}-\mathrm{of} \text { carboxamide moiety), } 7.57(\mathrm{~s}, 1 \mathrm{H} \text { of imidazole ring) and } 7.34-8.09(\mathrm{~m}, 4 \mathrm{H} \text {, } \\
\text { of phenoxy group). }\end{array}$ \\
\hline
\end{tabular}

Table 1.9: The ${ }^{13} \mathrm{C}$ NMR (75MHz) spectra of N-((6 - (4-chlorophenoxy) / (4-bromophenoxy) / (4-nitrophenoxy) - 6 - oxido - 4, 8-dihyro - $1 \mathrm{H}$ - [1,3,2] dioxaphophepino [5, 6-d] imidazole-1-yl-) methyl) morpholine - 4 carboxamide / piperidine - 1 - carboxamide / 4-methyl piperazine - 1 - carboxamide $11(\mathrm{a}-\mathrm{i})\left(\delta_{\mathrm{PPM}}\right)$ :

\begin{tabular}{|c|c|c|c|}
\hline COMP & $\mathrm{R}$ & $\mathrm{X}$ & ${ }^{13} \mathrm{C}-\mathrm{NMR}\left(\mathrm{DMSO}-\mathrm{d}_{6}\right)\left(\delta_{\mathrm{PPM}}\right)$ \\
\hline $11 \mathrm{a}$ & $\mathrm{Cl}$ & $\mathrm{O}$ & $\begin{array}{l}\text { 137.3, 132.9, 127.7, 63.0, 60.2, 62.7, 157.7, 46.6, 65.7, 148.3, 125.7, } 131.3 \text { and } 126.9 \\
\text { corresponding to } \mathrm{C}_{1}, \mathrm{C}_{2}, \mathrm{C}_{3}, \mathrm{C}_{4}, \mathrm{C}_{5}, \mathrm{C}_{6}, \mathrm{C}_{7}, \mathrm{C}_{8} \& \mathrm{C}_{11}, \mathrm{C}_{9} \& \mathrm{C}_{10}, \mathrm{C}_{12}, \mathrm{C}_{13} \& \mathrm{C}_{17}, \mathrm{C}_{14} \& \mathrm{C}_{16} \text { and } \mathrm{C}_{15} \text {. }\end{array}$ \\
\hline $11 \mathrm{~b}$ & $\mathrm{Cl}$ & $\mathrm{CH}_{2}$ & $\begin{array}{l}\text { 137.3, 132.9, 127.7, 63.0, 60.2, 62.7, 157.7, 49.3, 24.9, 23.8, 148.3, 125.7, } 131.3 \text { and } 126.9 \\
\text { corresponding to } \mathrm{C}_{1}, \mathrm{C}_{2}, \mathrm{C}_{3}, \mathrm{C}_{4}, \mathrm{C}_{5}, \mathrm{C}_{6}, \mathrm{C}_{7}, \mathrm{C}_{8} \& \mathrm{C}_{12}, \mathrm{C}_{9} \& \mathrm{C}_{11}, \mathrm{C}_{10}, \mathrm{C}_{13}, \mathrm{C}_{14} \& \mathrm{C}_{18}, \mathrm{C}_{15} \& \mathrm{C}_{17} \text { and } \\
\mathrm{C}_{16 .}\end{array}$ \\
\hline $11 \mathrm{c}$ & $\mathrm{Cl}$ & $\mathrm{N}-\mathrm{CH}_{3}$ & $\begin{array}{l}\text { 137.3, 132.9, 127.7, 63.0, 60.2, 62.7, 157.7, 51.7, 51.0, 46.6, 148.3, 125.7, } 131.3 \text { and } 126.9 \\
\text { corresponding to } \mathrm{C}_{1}, \mathrm{C}_{2}, \mathrm{C}_{3}, \mathrm{C}_{4}, \mathrm{C}_{5}, \mathrm{C}_{6}, \mathrm{C}_{7}, \mathrm{C}_{8} \& \mathrm{C}_{11}, \mathrm{C}_{9} \& \mathrm{C}_{10}, \mathrm{C}_{12}, \mathrm{C}_{13} \mathrm{C}_{14} \& \mathrm{C}_{18}, \mathrm{C}_{15} \& \mathrm{C}_{17} \text { and } \\
\mathrm{C}_{16 .}\end{array}$ \\
\hline $11 \mathrm{~d}$ & $\mathrm{Br}$ & $\mathrm{O}$ & $\begin{array}{l}\text { 137.3, 132.9, 127.7, 63.0, 60.2, 62.7, 157.7, 46.6, 65.7, 149.2, 123.0, } 133.3 \text { and } 115.7 \\
\text { corresponding to } C_{1}, C_{2}, C_{3}, C_{4}, C_{5}, C_{6}, C_{7}, C_{8} \& C_{11}, C_{9} \& C_{10},, C_{12}, C_{13} \& C_{17}, C_{14} \& C_{16} \text { and } C_{15} \text {. }\end{array}$ \\
\hline $11 \mathrm{e}$ & $\mathrm{Br}$ & $\mathrm{CH}_{2}$ & $\begin{array}{l}\text { 137.3, 132.9, 127.7, 63.0, 60.2, 62.7, 157.7, 49.3, 24.9, 23.8, 149.2, 123.0, } 133.3 \text { and } 115.7 \\
\text { corresponding to } \mathrm{C}_{1}, \mathrm{C}_{2}, \mathrm{C}_{3}, \mathrm{C}_{4}, \mathrm{C}_{5}, \mathrm{C}_{6}, \mathrm{C}_{7}, \mathrm{C}_{8} \& \mathrm{C}_{12}, \mathrm{C}_{9} \& \mathrm{C}_{11}, \mathrm{C}_{10}, \mathrm{C}_{13} \mathrm{C}_{14} \& \mathrm{C}_{18}, \mathrm{C}_{15} \& \mathrm{C}_{17} \text { and } \\
\mathrm{C}_{16 .}\end{array}$ \\
\hline $11 \mathrm{f}$ & $\mathrm{Br}$ & $\mathrm{N}-\mathrm{CH}_{3}$ & $\begin{array}{l}\text { 137.3, 132.9, 127.7, 63.0, 60.2, 62.7, 157.7, 51.7, 51.0, 46.6, 149.2, 123.0, 133.3 and } 115.7 \\
\text { corresponding to } \mathrm{C}_{1}, \mathrm{C}_{2}, \mathrm{C}_{3}, \mathrm{C}_{4}, \mathrm{C}_{5}, \mathrm{C}_{6}, \mathrm{C}_{7}, \mathrm{C}_{8} \& \mathrm{C}_{11}, \mathrm{C}_{9} \& \mathrm{C}_{10}, \mathrm{C}_{12}, \mathrm{C}_{13} \mathrm{C}_{14} \& \mathrm{C}_{18}, \mathrm{C}_{15} \& \mathrm{C}_{17} \text { and } \\
\mathrm{C}_{16 .}\end{array}$ \\
\hline $11 \mathrm{~g}$ & $\mathrm{NO}_{2}$ & $\mathrm{O}$ & $\begin{array}{l}\text { 137.3, 132.9, 127.7, 63.0, 60.2, 62.7, 157.7, 46.6, 65.7, 156.3, 121.9, } 126.3 \text { and 140.5corresponding } \\
\text { to } \mathrm{C}_{1}, \mathrm{C}_{2}, \mathrm{C}_{3}, \mathrm{C}_{4}, \mathrm{C}_{5}, \mathrm{C}_{6}, \mathrm{C}_{7}, \mathrm{C}_{8} \& \mathrm{C}_{11}, \mathrm{C}_{9} \& \mathrm{C}_{10}, \mathrm{C}_{12}, \mathrm{C}_{13} \& \mathrm{C}_{17}, \mathrm{C}_{14} \& \mathrm{C}_{16} \text { and } \mathrm{C}_{15} \text {. }\end{array}$ \\
\hline $11 \mathrm{~h}$ & $\mathrm{NO}_{2}$ & $\mathrm{CH}_{2}$ & $\begin{array}{l}\text { 137.3, 132.9, 127.7, 63.0, 60.2, 62.7, 157.7, 49.3, 24.9, 23.8, 156.3, 121.9, } 126.3 \text { and } 140.5 \\
\text { corresponding to } \mathrm{C}_{1}, \mathrm{C}_{2}, \mathrm{C}_{3}, \mathrm{C}_{4}, \mathrm{C}_{5}, \mathrm{C}_{6}, \mathrm{C}_{7}, \mathrm{C}_{8} \& \mathrm{C}_{12}, \mathrm{C}_{9} \& \mathrm{C}_{11}, \mathrm{C}_{10}, \mathrm{C}_{13} \mathrm{C}_{14} \& \mathrm{C}_{18}, \mathrm{C}_{15} \& \mathrm{C}_{17} \text { and } \\
\mathrm{C}_{16 .}\end{array}$ \\
\hline $11 \mathrm{i}$ & $\mathrm{NO}_{2}$ & $\mathrm{~N}-\mathrm{CH}_{3}$ & $\begin{array}{l}\text { 137.3, 132.9, 127.7, 63.0, 60.2, 62.7, 157.7, 51.7, 51.0, 46.6, 156.3, 121.9, 126.3 and } 140.5 \\
\text { corresponding to } \mathrm{C}_{1}, \mathrm{C}_{2}, \mathrm{C}_{3}, \mathrm{C}_{4}, \mathrm{C}_{5}, \mathrm{C}_{6}, \mathrm{C}_{7}, \mathrm{C}_{8} \& \mathrm{C}_{11}, \mathrm{C}_{9} \& \mathrm{C}_{10}, \mathrm{C}_{12}, \mathrm{C}_{13} \mathrm{C}_{14} \& \mathrm{C}_{18}, \mathrm{C}_{15} \& \mathrm{C}_{17} \text { and } \\
\mathrm{C}_{16 .}\end{array}$ \\
\hline
\end{tabular}


Table 1.10: The ${ }^{31} \mathrm{P}$ NMR (161.89MHz) spectra of N-((6 - (4-chlorophenoxy) / (4-bromophenoxy) / (4nitrophenoxy) - 6 - oxido - 4, 8-dihyro - 1H - [1,3,2] dioxaphophepino [5, 6-d] imidazole-1-yl-) methyl) morpholine - 4 - carboxamide / 4-methyl piperazine - 1 - carboxamide $(11 \mathrm{a}, 11 \mathrm{c}, 11 \mathrm{~d}, 11 \mathrm{f}, 11 \mathrm{~g}$ and $11 \mathrm{i})\left(\delta_{\mathrm{PPM}}\right)$ :

\begin{tabular}{|c|c|c|c|}
\hline COMP & $\mathrm{R}$ & $\mathrm{X}$ & ${ }^{31} \mathrm{P}-\mathrm{NMR}\left(\mathrm{DMSO}-\mathrm{d}_{6}\right)\left(\delta_{\text {PPM }}\right)$ \\
\hline $11 \mathrm{a}$ & $\mathrm{Cl}$ & $\mathrm{O}$ & -7.30 and -11.10 \\
\hline $11 \mathrm{c}$ & $\mathrm{Cl}$ & $\mathrm{N}-\mathrm{CH}_{3}$ & -7.50 \\
\hline $11 \mathrm{~d}$ & $\mathrm{Br}$ & $\mathrm{O}$ & -7.70 and -11.40 \\
\hline $11 \mathrm{f}$ & $\mathrm{Br}$ & $\mathrm{O}$ & -7.80 \\
\hline $11 \mathrm{~g}$ & $\mathrm{NO}_{2}$ & $\mathrm{O}$ & -6.90 and -10.80 \\
\hline $11 \mathrm{i}$ & $\mathrm{NO}_{2}$ & $\mathrm{~N}-\mathrm{CH}_{3}$ & -7.20 \\
\hline
\end{tabular}

Table 1.11: Pysical and Analytical data of N-((6 - (4-chlorophenoxy) / (4-bromophenoxy) / (4-nitrophenoxy) - 6 - oxido - 4, 8-dihyro - 1H - [1, 3, 2] dioxaphophepino [5, 6-d] imidazole-1-yl-) methyl) morpholine - 4 carboxamide / piperidine - 1 - carboxamide / 4-methyl piperazine - 1 - carboxamide 11(a-i):

\begin{tabular}{|c|c|c|c|c|c|c|c|}
\hline \multirow[t]{2}{*}{ COMP } & \multirow[t]{2}{*}{$\begin{array}{l}\text { MOLECULAR } \\
\text { FORMULA }\end{array}$} & \multirow[t]{2}{*}{$\operatorname{MP}\left({ }^{0} \mathrm{C}\right)$} & \multirow[t]{2}{*}{$\begin{array}{l}\text { YIELD } \\
(\%)\end{array}$} & \multicolumn{4}{|c|}{$\begin{array}{l}\text { ELEMENTAL } \\
\text { ANALYSS(\%) }\end{array}$} \\
\hline & & & & & DUND & & ALC \\
\hline $11 \mathrm{a}$ & $\mathrm{C}_{17} \mathrm{H}_{20} \mathrm{ClN}_{4} \mathrm{O}_{6} \mathrm{P}$ & $152-155$ & 70 & $\begin{array}{l}\mathrm{C} \\
\mathrm{H} \\
\mathrm{Cl} \\
\mathrm{N} \\
\mathrm{P}\end{array}$ & $\begin{array}{c}45.31, \\
4.05, \\
7.21, \\
12.05, \\
6.30 .\end{array}$ & $\begin{array}{l}\mathrm{C} \\
\mathrm{H} \\
\mathrm{C}\end{array}$ & $\begin{array}{c}46.11, \\
4.55, \\
8.01, \\
12.65, \\
7.00 .\end{array}$ \\
\hline $11 b$ & $\mathrm{C}_{18} \mathrm{H}_{22} \mathrm{ClN}_{4} \mathrm{O}_{5} \mathrm{P}$ & $133-136$ & 67 & $\begin{array}{l}\mathrm{C} \\
\mathrm{H} \\
\mathrm{Cl} \\
\mathrm{N} \\
\mathrm{P}\end{array}$ & $\begin{array}{c}48.24, \\
4.53, \\
7.24, \\
12.11, \\
6.33 .\end{array}$ & $\begin{array}{l}\mathrm{C} \\
\mathrm{H} \\
\mathrm{C}\end{array}$ & $\begin{array}{c}49.04, \\
5.03, \\
8.04, \\
12.71, \\
7.03 .\end{array}$ \\
\hline $11 \mathrm{c}$ & $\mathrm{C}_{18} \mathrm{H}_{23} \mathrm{ClN}_{5} \mathrm{O}_{5} \mathrm{P}$ & $172-175$ & 65 & $\begin{array}{l}\mathrm{C} \\
\mathrm{H} \\
\mathrm{Cl} \\
\mathrm{N} \\
\mathrm{P}\end{array}$ & $\begin{array}{r}46.63, \\
4.59, \\
6.98, \\
14.76, \\
6.09 .\end{array}$ & $\begin{array}{l}\mathrm{C} \\
\mathrm{H} \\
\mathrm{C}\end{array}$ & $\begin{array}{r}47.43, \\
5.09, \\
7.78, \\
15.36, \\
79 .\end{array}$ \\
\hline $11 \mathrm{~d}$ & $\mathrm{C}_{17} \mathrm{H}_{20} \mathrm{BrN}_{4} \mathrm{O}_{6} \mathrm{P}$ & $146-149$ & 75 & $\begin{array}{l}\mathrm{C} \\
\mathrm{H} \\
\mathrm{Br} \\
\mathrm{N} \\
\mathrm{P}\end{array}$ & $\begin{array}{l}49.11, \\
3.64, \\
15.80, \\
10.90, \\
5.66 .\end{array}$ & $\begin{array}{l}\mathrm{C} \\
\mathrm{H} \\
\mathrm{B} \\
\mathrm{N} \\
\mathrm{P}\end{array}$ & $\begin{array}{c}49.91, \\
4.14, \\
16.40, \\
11.50, \\
6.36 .\end{array}$ \\
\hline $11 \mathrm{e}$ & $\mathrm{C}_{18} \mathrm{H}_{22} \mathrm{BrN}_{4} \mathrm{O}_{5} \mathrm{P}$ & $165-168$ & 70 & $\begin{array}{l}\mathrm{C} \\
\mathrm{H} \\
\mathrm{Br} \\
\mathrm{N} \\
\mathrm{P} \\
\end{array}$ & $\begin{array}{r}43.75, \\
4.07, \\
587, \\
10.95, \\
5.68 .\end{array}$ & $\begin{array}{l}\mathrm{C} \\
\mathrm{H} \\
\mathrm{B} \\
\mathrm{N} \\
\mathrm{P}\end{array}$ & $\begin{array}{c}44.55, \\
4.57, \\
16.47, \\
11.55, \\
6.38 .\end{array}$ \\
\hline $11 \mathrm{f}$ & $\mathrm{C}_{18} \mathrm{H}_{23} \mathrm{BrN}_{5} \mathrm{O}_{5} \mathrm{P}$ & $189-192$ & 68 & $\begin{array}{l}\mathrm{C} \\
\mathrm{H} \\
\mathrm{Br} \\
\mathrm{N} \\
\mathrm{P}\end{array}$ & $\begin{array}{l}42.41, \\
4.13, \\
15.37, \\
13.40, \\
5.49 .\end{array}$ & $\begin{array}{l}\mathrm{C} \\
\mathrm{H} \\
\mathrm{B} \\
\mathrm{N} \\
\mathrm{P}\end{array}$ & $\begin{array}{c}43.21, \\
4.63, \\
15.97, \\
14.00, \\
6.19 .\end{array}$ \\
\hline $11 \mathrm{~g}$ & $\mathrm{C}_{17} \mathrm{H}_{20} \mathrm{~N}_{5} \mathrm{O}_{8} \mathrm{P}$ & $180-183$ & 65 & $\begin{array}{l}\mathrm{C} \\
\mathrm{H} \\
\mathrm{N} \\
\mathrm{P} \\
\end{array}$ & $\begin{array}{r}44.24, \\
3.95, \\
14.85, \\
6.13 .\end{array}$ & $\begin{array}{l}\mathrm{C} \\
\mathrm{H} \\
\mathrm{N} \\
\mathrm{P}\end{array}$ & $\begin{array}{r}45.04, \\
4.45, \\
15.45, \\
6.83 .\end{array}$ \\
\hline $11 \mathrm{~h}$ & $\mathrm{C}_{18} \mathrm{H}_{22} \mathrm{~N}_{5} \mathrm{O}_{7} \mathrm{P}$ & $177-180$ & 60 & $\begin{array}{l}\mathrm{C} \\
\mathrm{H} \\
\mathrm{N} \\
\mathrm{P}\end{array}$ & $\begin{array}{c}47.10, \\
4.41, \\
14.92, \\
6.16 .\end{array}$ & $\begin{array}{l}\mathrm{C} \\
\mathrm{H} \\
\mathrm{N} \\
\mathrm{P}\end{array}$ & $\begin{array}{c}47.90, \\
4.91, \\
15.52, \\
6.86 .\end{array}$ \\
\hline $11 \mathrm{i}$ & $\mathrm{C}_{18} \mathrm{H}_{23} \mathrm{~N}_{6} \mathrm{O}_{7} \mathrm{P}$ & 206-209 & 68 & $\begin{array}{l}\mathrm{C} \\
\mathrm{H} \\
\mathrm{N} \\
\mathrm{P}\end{array}$ & $\begin{array}{r}45.55, \\
4.47, \\
17.42, \\
5.94 .\end{array}$ & $\begin{array}{l}\mathrm{C} \\
\mathrm{H} \\
\mathrm{N} \\
\mathrm{P} \\
\end{array}$ & $\begin{array}{c}46.35, \\
4.97, \\
18.02, \\
6.64 .\end{array}$ \\
\hline
\end{tabular}

\section{Biological activity}

The antimicrobial activity [16] of these newly synthesized compounds was performed according to disc diffusion method, as recommended by the National Committee for Clinical Laboratory. The synthesised compounds were used at the concentration of $250 \mu \mathrm{g} / \mathrm{ml} \mathrm{DMF}$ as a solvent [17].

\section{Anti-bacterial activity}

The antibacterial activity of Imidazole Ureas / Carboxamides containing Dioxaphospholanes 7(a-l) and 11(a-i) were screened against the Staphylococcus aureus and Bacillus cerus (gram positive) and Escherichia coli, Pseudomonasaeruginosa (gram negative) organisms. Most of the compounds exhibited good antibacterial 
activity against both bacteria. The presence of nitro (7j, 7k) and (11g, 11i), chloro (7c, 7d) and (11a, 11c) and bromo (7h, 11d) were showed more activity than other substituted compounds. Here Amoxicillin is tested as reference compound to compare the activity. The anti-bacterial activity was shown in the Table $\mathbf{1 . 1 2}$

Table I.12: Anti-bacterial activity (Diameter zone of Inhibition in mm) of compounds of 7(a-1) and 11(a-i) (250 $\mu \mathrm{g} / \mathrm{ml})$ :

\begin{tabular}{|c|c|c|c|c|c|}
\hline \multirow[b]{2}{*}{ S.NO } & \multirow[b]{2}{*}{ COMP NO } & \multicolumn{4}{|c|}{ Zone of inhibition $(\mathrm{mm})$} \\
\hline & & $\begin{array}{c}\text { Staphylococcus aureus } \\
\text { NCCS } 2079 \\
250(\mu \mathrm{g} / \text { disc })\end{array}$ & $\begin{array}{c}\text { Bacillus cereus } \\
\text { NCCS } 2106 \\
250(\mu \mathrm{g} / \mathrm{disc})\end{array}$ & $\begin{array}{c}\text { Escherichia coli } \\
\text { NCCS } 2065 \\
250(\mu \mathrm{g} / \mathrm{dis})\end{array}$ & $\begin{array}{c}\text { Pseudomonas } \\
\text { aeruginosa } \\
\text { NCCS } 2200 \\
250(\mu \mathrm{g} / \text { disc }) \\
\end{array}$ \\
\hline 1 & $7 \mathrm{a}$ & 12 & 16 & 15 & 14 \\
\hline 2 & $7 \mathrm{~b}$ & 13 & 17 & 16 & 15 \\
\hline 3 & $7 \mathrm{c}$ & 15 & 19 & 18 & 17 \\
\hline 4 & $7 \mathrm{~d}$ & 16 & 20 & 19 & 18 \\
\hline 5 & $7 \mathrm{e}$ & 09 & 13 & 12 & 11 \\
\hline 6 & $7 \mathrm{f}$ & 10 & 14 & 13 & 12 \\
\hline 7 & $7 \mathrm{~g}$ & 12 & 16 & 15 & 14 \\
\hline 13 & $11 \mathrm{a}$ & 15 & 19 & 18 & 17 \\
\hline 14 & $11 \mathrm{~b}$ & 11 & 15 & 14 & 13 \\
\hline 15 & $11 \mathrm{c}$ & 14 & 18 & 17 & 16 \\
\hline 16 & $11 \mathrm{~d}$ & 12 & 16 & 15 & 14 \\
\hline 17 & $11 \mathrm{e}$ & 09 & 13 & 12 & 11 \\
\hline 18 & $11 \mathrm{f}$ & 10 & 14 & 13 & 12 \\
\hline 19 & $11 \mathrm{~g}$ & 16 & 20 & 19 & 18 \\
\hline 20 & $11 \mathrm{~h}$ & 13 & 17 & 16 & 15 \\
\hline 21 & $11 \mathrm{i}$ & 14 & 18 & 17 & 16 \\
\hline \multicolumn{2}{|c|}{ Amoxiciilin } & 21 & 27 & 24 & 22 \\
\hline
\end{tabular}

\section{Anti-fungal activity}

Antifungal activity of Imidazole Ureas / Carboxamides containing Dioxaphospholanes 7(a-l) and 11(ai) were screened against Aspergillus niger and Candida albicans. Most of the compounds exhibit good antifungal activity against both fungai. Most of the compounds exhibit good antifungal activity against both fungai. The presence of nitro $(\mathbf{7 j}, 7 \mathbf{k})$ and $(\mathbf{1 1 g}, \mathbf{1 1 i})$ chloro $(\mathbf{7 c}, \mathbf{7 d})$ and $(\mathbf{1 1 a}, \mathbf{1 1 c})$ and bromo $(\mathbf{7 h}, \mathbf{1 1 d})$ were showed more activity than other substituted compounds. Here Ketoconazole is tested as reference compound to compare the activity. The anti-fungalactivity was shown in the Table 1.13.

Table I.13: Anti-bacterial activity (Diameter zone of Inhibition in $\mathrm{mm}$ ) of compounds of 7(a-1) and 11(a-i) $(250 \mu \mathrm{g} / \mathrm{ml})$ :

\begin{tabular}{|c|c|c|c|}
\hline \multirow{2}{*}{ S.NO } & \multirow{2}{*}{ COMP NO } & $\begin{array}{c}|c| \\
\text { Aspergillus niger } \\
\text { NCCS } 1196 \\
250(\mu \mathrm{g} / \mathrm{disc})\end{array}$ & $\begin{array}{c}\text { Candida albicans } \\
\text { NCCS } 3471 \\
250(\mu \mathrm{g} / \mathrm{disc})\end{array}$ \\
\hline 1 & $7 \mathrm{a}$ & 09 & 07 \\
\hline 2 & $7 \mathrm{~b}$ & 10 & 08 \\
\hline 3 & $7 \mathrm{c}$ & 12 & 10 \\
\hline 4 & $7 \mathrm{~d}$ & 16 & 14 \\
\hline 5 & $7 \mathrm{e}$ & 08 & 06 \\
\hline 6 & $7 \mathrm{f}$ & 10 & 08 \\
\hline 7 & $7 \mathrm{~g}$ & 12 & 10 \\
\hline 8 & $7 \mathrm{~h}$ & 14 & 12 \\
\hline 9 & $7 \mathrm{i}$ & 13 & 11 \\
\hline 10 & $7 \mathrm{j}$ & 15 & 13 \\
\hline 11 & $7 \mathrm{k}$ & 17 & 15 \\
\hline 12 & 71 & 19 & 17 \\
\hline 13 & $11 \mathrm{a}$ & 17 & 15 \\
\hline 14 & $11 \mathrm{~b}$ & 12 & 10 \\
\hline 15 & $11 \mathrm{c}$ & 15 & 13 \\
\hline 16 & $11 \mathrm{~d}$ & 14 & 12 \\
\hline 17 & $11 \mathrm{e}$ & 09 & 07 \\
\hline 18 & $11 \mathrm{f}$ & 12 & 10 \\
\hline 19 & $11 \mathrm{~g}$ & 20 & 18 \\
\hline 20 & $11 \mathrm{~h}$ & 16 & 14 \\
\hline 21 & $11 \mathrm{i}$ & 18 & 16 \\
\hline \multicolumn{2}{|r|}{ Ketoconazole } & 22 & 25 \\
\hline
\end{tabular}




\section{Conclusions}

The newly synthesized compounds Imidazole Ureas / Carboxamides containing Dioxaphospholanes derivatives $7(\mathrm{a}-1)$ and $11(\mathrm{a}-\mathrm{i})$ were found to be active in the study of anti-bacterial and anti-fungal activity. It can be concluded that this class of compounds certainly holds great promise towards the pursuit to discover novel classes of antimicrobial agents.

\section{Acknowledgement}

The authors (V.E.R and CH.L.P) thanks to U G C - S A P and U G C - B S R, New Delhi for financial assistance. They are also thankful to IICT Hyderabad and CDRI Lucknow for spectral and analytical data.

\section{References}

[1] P.k.Naithani, V.K.Srivastava, J.P.Bharathwal, A.K. Saxena, T.K.Gupta and K.Shankhar, Indian J.Chem, 28B, 1989, 229

[2] M. Harfenist, E.F.Soroko and G.M.Mckenzie, J.Med. chem, 21, 1978 , 405. [3]A.R.KatritzkyComphrehensive Heterocyclic Chemistry, Vol 5, 1984, P. 497-98

[4] Sridevi kona,Venkata N.R Chava, Rama Suresh Ravi, Ramu Sridhar P and manab Chakravarthy, Der chemica Sinica, 3 (3), 2012 , 548-556.

[5] E. Breuer, The chemistry of organophosphorus compounds, Heartly, F. R. Ed, John wiely and sons, Newyork, Vol 4, 1996,653

[6] Faraci, W.S., Yang, B .V., D.O, Rourke , R.W.Spencer ., Bioorg. Med. Chem., 3,1995, 605.

[7] Nivarkar, M. Gupta, A. K. Kaushik, M.P., Tetrahedron Lett., 45, 2004, 6863.

[8] Y. Haranadha Reddy, B. Siva kumar, G. Chandrashekar Reddy, E. Dadapear and K.Subramanyam Reedy, Der chemica Sinica, 3 (4), 2012, 817-823

[9] Mehellou, Y., MeGuigan, C., Brancale, A., Balzarini, J., Bioorg.Med.chem.Lett, 17, 2007, 3666.

[10] Guigan, C.M., Thiery, J .C., Daverio, F., Jiang, W. J., Da-vies , G., Mason, M., Bioorg. Med. Chem, 13, $2005,3219$.

[11] A M Polozov, AV Khotinen and EN Klimovitskii, phosphorus, sulphur, silicon Relat, Elem, 581,1996, 109-110.

[12] M K Rubtsova and R D Zhilina, ZhurPriklad Khim, 32, 2604, 1959 ; Chem Abstr, 54, 1960, 8683f.

[13] X Francis, FX Markley and CJ Worrel, US pat, 3153081; Chem Abstr, 62, 1965, 483.

[14] Basanagoud S.Patil, Ganga-Ramu Vasanthakumar and Vommina V.Suresh Babu, J.Org.Chem.,68, 2003, 7274-7280.

[15] Khiangte Vanladinpuia, Ghanashyam Bez* Tetrahedron Letters 52, 2011, 3759-3764.

[16] Arun K. Wahi and Arti Singh, Der ChemicaSinica, 2 (3), 2011, 11-19.

[17] Sudhir Bharadwaj, Bharat Parashar, NarendraParashar and V.K.Sharma, Scholar Research Library, Achieves of Applied Science Research, 3(2), 2011, 558-567. 